\title{
Effect of homogeneous and heterogeneous reactions on the solute dispersion in composite porous medium
}

\author{
J. Prathap Kumar, J.C. Umavathi* and Shivakumar Madhavarao \\ Department of Mathematics, Gulbarga University, Gulbarga-585 106, Karnataka, INDIA. \\ *Corresponding author email: jc_uma11@yahoo.com
}

\begin{abstract}
The Taylor dispersion of a solute for composite porous medium between two parallel plates with first-order chemical reaction is studied analytically. The fluids in both the regions are incompressible and the transport properties are assumed to be constant. The results are shown graphically for various values of viscosity ratio, pressure gradient and porous parameter on the effective dispersion coefficient and volumetric flow rate. It is found that for both homogeneous and heterogeneous chemical reactions, the effective dispersion coefficient decreases as porous parameter increases. The validity of the results obtained for composite porous media is verified by comparison with the available porous medium results for one fluid model and good agreement is found. Also two fluid model in the absence of porous matrix is compared with the available one fluid model results and the values tally very well.
\end{abstract}

Keywords: Taylor dispersion, immiscible fluids, horizontal channel, porous medium, chemical reaction.

DOI: http://dx.doi.org/10.4314/ijest.v4i2.5

\section{Introduction}

Flow through and past porous media has attracted considerable interest in recent years because of its importance in science, engineering and technology (see Kaviany, 1999 and Vafai, 2000). Notable practical problems that require the study of DIPM (Dispersion In Porous Media) are the extraction of energy from geothermal regions, analyzing thermal energy storage systems, thermal insulation, solar collectors with porous absorbers, biomechanical applications such as cartilage in synovial joints (see Sueiu et al., 2003; Ng et al., 2005), pollutant transport resulting from municipal, industrial, and agricultural wastes through the surface of the earth, polluting ground water where control of pollutants is desired and the tiny dust particles floating in the air, known as aerosols and which are gradually choking people to death, where an understanding of the spreading of aerosols is required. The main interest in all these cases is the study of dispersion (i.e., spreading leading to flow-enhanced diffusion) in porous media. Chemical reactions can be classified as either homogenous or heterogeneous processes. A homogeneous reaction is one that occurs uniformly through a given phase. In contrast, a heterogeneous reaction takes place in a restricted region or within the boundary of a phase. A reaction is said to be the first order if the rate of reaction is directly proportional to the concentration itself. In many chemical engineering processes, a chemical reaction between a foreign mass and the fluid does occur. However, the diffusion process, combined with homogeneous and heterogeneous chemical reactions of a solute in a porous medium, is also important in chemical engineering. The diffusion inside the tortuous void passages in the medium is pointed out by Bird et al. (1960).

These problems of dispersion have been receiving considerable attention from chemical, environmental, petroleum engineers, hydrologists, mathematicians and soil scientists. Most of the works reveal common assumptions of homogeneous porous media with constant porosity, steady seepage flow velocity and constant dispersion coefficient. For such assumptions Hoopes and Harleman (1965) studied the problem of dispersion in radial flow from fully penetrating, homogeneous, isotropic non-adsorbing confined aquifer. Bruce and Street (1966) considered both longitudinal and lateral dispersion with in semi-infinite no adsorbing porous media in a steady unidirectional flow field for a constant input concentration. Marino (1974) considered the input 
concentration varying exponentially with time. Al-Niami and Rushton (1977) and Marino (1978) studied the analysis of flow against dispersion in porous media. The influences of thermal diffusion and magnetic field on combined free-forced convection and mass transfer flow past a vertical porous flat plate, in the presence of heat generation were studied numerically by AbdelRahman (2008).

The permeabilities for porous media, both saturated and unsaturated, have received much attention (Kaviany, 1999; Panfilov, 2000) due to practical applications, including chemical engineering, soil science and engineering. Compared with single-phase (or saturated) flow in porous media, the multiphase (or unsaturated) immiscible flows in porous media are not well understood. The multiphase immiscible flows in porous media are very important in practical applications such as the petroleum industry, chemical engineering, and soil engineering. In order to get a better understanding of the mechanisms for permeability, the analytical solution for permeability of porous media becomes a challenging task. Katz and Thompson (1985) presented experimental evidence indicating that the pore spaces of a set of porous sandstone samples (in nature) are fractals and are self-similar over 3 to 4 orders of magnitude in length extending from $10 \AA$ to $100 \mu \mathrm{m}$. They argued that the pore volume is a fractal with the same fractal dimension as the pore-rock interface. According to the fractal character of real porous media, Yu and Cheng (2002) developed a fractal permeability model for bidispersed saturated porous media, and this fractal model is also applicable to porous fabrics (Yu et al., 2002) (see Figure 4; Yu et al., 2001). A fractal analysis of permeabilities for porous media, both saturated and unsaturated, was presented by Boming and Wie (2004) based on the fractal nature of pores in the media. Yongting et al. (2003) studied the selfsimilarity model for effective thermal conductivity of porous media. Recently Boming (2008) analyzed the flow in fractal porous media.

Fluid flow and heat transfer characteristics at the interface region in systems which consists of a fluid-saturated porous medium and an adjacent horizontal fluid layer received considerable attention. Poulikakos et al. (1986) studied the conduction for composite system consisting of a fluid layer and packed bed heated from below. Beckermann et al. $(1987,1988)$ also made a deep analysis of heat transfer with various positions allocated for porous/fluid layers. Prasad (1991) have made an excellent review for composite flow systems. The problem of boundary conditions at the porous medium/clear fluid interface was first investigated in Beavers and Joseph (1967). Neale and Nader (1974) presented one of the earlier attempts regarding this type of boundary condition in porous medium. In this study the authors proposed the continuity in both the velocity and the velocity gradient at the interface by introducing the Brinkman term in the momentum equation for the porous side. Vafai and Kim (1990) presented an exact solution for the fluid flow with the interface between a porous medium and a fluid layer, including the inertia and boundary effects. For more details on interfacial conditions, readers are referred to the paper by Alzami and Vafai (2001). Following the analysis of Neale and Nader (1974), Malashetty et al. (2001, 2005), Umavathi et al. (2006, 2009, 2010) and Prathap Kumar et al. (2009, 2010) studied flow and heat transfer of composite porous medium. The combined effects of homogeneous and heterogeneous chemical reaction for a solute dispersing in Newtonian fluid flow have been discussed by Walker (1961), Soloman and Hudson (1967), Gupta and Gupta (1972) and others. Recently Rudraiah and Ng (2007) reviewed the dispersion in porous media with and without chemical reaction.

The literature on hydrodynamic dispersion in porous medium is very sparse in spite of its versatile applications in many branches of sciences, engineering and technology. Therefore the objective of this work is to present a mechanism of dispersion with chemical reaction in a channel filled with immiscible fluid saturated porous medium with the motive that the results obtained may be effectively used in some typical cases to understand the mechanism of mixing and separation process. Further this work also provides an improved model for dispersion in porous medium that agrees with the experimental data of Harlemann et al. (1963).

\section{Mathematical formulation of the problem}

The physical configuration considered in this study is shown in Figure 1. Consider the laminar flow of two immiscible fluids saturated with porous medium between two parallel plates distant $2 h$ apart, taking $X$-axis along the mid-section of the channel and $Y$-axis perpendicular to the walls. Region-1 $(-h \leq Y \leq 0)$ is filled with the fluid saturated porous medium of density $\rho_{1}$, viscosity $\mu_{1}$, permeability $\kappa_{1}$ under a uniform pressure gradient $\frac{d P_{1}}{d X}$ whereas region-2 $(0 \leq Y \leq h)$ is filled with another fluid saturated with different porous medium of density $\rho_{2}$, viscosity $\mu_{2}$, permeability $\kappa_{2}$ under a uniform pressure gradient $\frac{d P_{2}}{d X}$. The fluids in both the regions are Newtonian fluids.

It is assumed that the flow is steady, laminar, fully developed, and that fluid properties are constant. The flow in both regions is assumed to be driven by a common constant pressure gradient. Under these assumptions, the governing equations of motion for incompressible fluids are (Neale and Nader, 1974)

Region-1

$$
\frac{d^{2} U_{1}}{d Y^{2}}-\frac{1}{\kappa_{1}} U_{1}=\frac{1}{\mu_{1}} \frac{d P_{1}}{d X}
$$


Region-2

$$
\frac{d^{2} U_{2}}{d Y^{2}}-\frac{1}{\kappa_{2}} U_{2}=\frac{1}{\mu_{2}} \frac{d P_{2}}{d X}
$$

where $U_{i}$ is the $X$-component of fluid velocity and $P_{i}$ is the pressure. The subscripts 1 and 2 denote the values for region-1 and region-2 respectively.



Figure 1: Physical configuration

The boundary conditions on velocity are no-slip conditions requiring that the velocity must vanish at the walls. In addition, continuity of velocity and shear stress at the interface is assumed. With these assumptions, the boundary and interface conditions on velocity become

$$
\begin{array}{cccc}
U_{1}=0 \quad \text { at } & Y=-h ; & U_{2}=0 \quad \text { at } \quad Y=h & \\
U_{1}=U_{2} \text { at } & Y=0 ; & \mu_{1} \frac{d U_{1}}{d Y}=\mu_{2} \frac{d U_{2}}{d Y} \quad \text { at } \quad Y=0
\end{array}
$$

Using the non-dimensional parameters

$$
\eta=\frac{Y}{h}, u_{1}=\frac{\rho_{1} h}{\mu_{1}} U_{1}, u_{2}=\frac{\rho_{2} h}{\mu_{2}} U_{2}, x=\frac{X}{h}, p_{1}^{*}=\frac{P_{1}}{\rho_{1}\left(v_{1} / h\right)^{2}}, p_{2}^{*}=\frac{P_{2}}{\rho_{2}\left(v_{2} / h\right)^{2}}, \sigma_{i}=\frac{h}{\sqrt{\kappa_{i}}}
$$

in the Eqs. (1) to (3) become

$$
\begin{gathered}
\frac{d^{2} u_{1}}{d \eta^{2}}-\sigma_{1}^{2} u_{1}=p_{1} \\
\frac{d^{2} u_{2}}{d \eta^{2}}-\sigma_{2}^{2} u_{2}=p_{2} \\
u_{1}=0 \quad \text { at } \quad \eta=-1 ; \quad u_{2}=0 \quad \text { at } \quad \eta=1 \\
u_{1}=m n u_{2} ; \quad \frac{d u_{1}}{d \eta}=m^{2} n \frac{d u_{2}}{d \eta} \text { at } \quad \eta=0
\end{gathered}
$$

where $\quad p_{1}=\frac{d p_{1}^{*}}{d x}, p_{2}=\frac{d p_{2}^{*}}{d x}, m=\mu_{2} / \mu_{1}$ and $n=\rho_{1} / \rho_{2}$

Solutions of Eqs. (5) and (6) are

$$
\begin{aligned}
& u_{1}=a_{1} \cosh \left(\sigma_{1} \eta\right)+a_{2} \sinh \left(\sigma_{1} \eta\right)-\frac{p_{1}}{\sigma_{1}^{2}} \\
& u_{2}=a_{3} \cosh \left(\sigma_{2} \eta\right)+a_{4} \sinh \left(\sigma_{2} \eta\right)-\frac{p_{2}}{\sigma_{2}^{2}}
\end{aligned}
$$

where $a_{1}, a_{2}, a_{3}$ and $a_{4}$ are integrating constants that are evaluated by using boundary and interface conditions as given in Eqn. (7).

From Eqs. (8) and (9) the average velocities become

$$
\bar{u}_{1}=\frac{1}{2} \int_{-1}^{0} u_{1} d \eta=\frac{a_{2}}{2 \sigma_{1}}+\frac{a_{1}}{2 \sigma_{1}} \sinh \left(\sigma_{1}\right)-\frac{a_{2}}{2 \sigma_{1}} \cosh \left(\sigma_{1}\right)-\frac{p_{1}}{2 \sigma_{1}^{2}}
$$




$$
\bar{u}_{2}=\frac{1}{2} \int_{0}^{1} u_{2} d \eta=\frac{a_{3}}{2 \sigma_{2}} \sinh \left(\sigma_{2}\right)+\frac{a_{4}}{2 \sigma_{2}} \cosh \left(\sigma_{2}\right)-\frac{p_{2}}{2 \sigma_{2}^{2}}-\frac{a_{4}}{2 \sigma_{2}}
$$

\section{Case 1: Diffusion with a homogeneous first-order chemical reaction}

We assume that a solute diffuses and simultaneously undergoes first-order irreversible chemical reaction in the liquid under isothermal conditions. The equation for the concentration $C_{1}$ of the solute for the region-1 satisfies

$$
\frac{\partial C_{1}}{\partial t}+u_{1} \frac{\partial C_{1}}{\partial X}=D_{1}\left(\frac{\partial^{2} C_{1}}{\partial X^{2}}+\frac{\partial^{2} C_{1}}{\partial Y^{2}}\right)-K_{1} C_{1}
$$

Similarly, the equation for the concentration $C_{2}$ of the solute for the region-2 satisfies

$$
\frac{\partial C_{2}}{\partial t}+u_{2} \frac{\partial C_{2}}{\partial X}=D_{2}\left(\frac{\partial^{2} C_{2}}{\partial X^{2}}+\frac{\partial^{2} C_{2}}{\partial Y^{2}}\right)-K_{2} C_{2}
$$

in which $D_{1}$ and $D_{2}$ are the molecular diffusion coefficients (assumed constants) for the region-1 and region-2 respectively and $K_{1}$ and $K_{2}$ are the first-order reaction rate constant. In deriving the above Eqs. (12) and (13), it is assumed that the solute is present in a small concentration, the last term $-\mathrm{K}_{1} \mathrm{C}_{1} / \mathrm{mol} \mathrm{m}^{-3} \mathrm{~s}^{-1}$ and $-\mathrm{K}_{2} \mathrm{C}_{2} / \mathrm{mol} \mathrm{m}^{-3} \mathrm{~s}^{-1}$ represents the volume rate of disappearance of the solute due to chemical reaction. We now assume that

$$
\frac{\partial^{2} C_{1}}{\partial X^{2}}<<\frac{\partial^{2} C_{1}}{\partial Y^{2}} \text { and } \frac{\partial^{2} C_{2}}{\partial X^{2}}<<\frac{\partial^{2} C_{2}}{\partial Y^{2}}
$$

If we now consider convection across a plane moving with the mean speed of the flow, then relative to this plane the fluid velocities are given by

Region-1

Region-2

$$
u_{1 x}=u_{1}-\bar{u}=a_{1} \cosh \left(\sigma_{1} \eta\right)+a_{2} \sinh \left(\sigma_{1} \eta\right)+l_{1}
$$

$$
u_{2 x}=u_{2}-\bar{u}=a_{3} \cosh \left(\sigma_{2} \eta\right)+a_{4} \sinh \left(\sigma_{2} \eta\right)+l_{2}
$$

where $\bar{u}$ is the sum of average velocities of region-1 and region-2.

Introducing the dimensionless quantities

$$
\theta_{1}=\frac{t_{1}}{\bar{t}_{1}}, \bar{t}_{1}=\frac{L_{1}}{\bar{u}_{1}}, \xi_{1}=\frac{x_{1}-\bar{u}_{1} t_{1}}{L_{1}}, \theta_{2}=\frac{t_{2}}{\bar{t}_{2}}, \bar{t}_{2}=\frac{L_{2}}{\bar{u}_{2}}, \xi_{2}=\frac{x_{2}-\bar{u}_{2} t_{2}}{L_{2}}
$$

and using Eqs. (14) and (15), the Eqs. (12) and (13) become

Region-1

$$
\frac{1}{t} \frac{\partial C_{1}}{\partial \theta_{1}}+\frac{u_{1 x}}{L_{1}} \frac{\partial C_{1}}{\partial \xi_{1}}=\frac{D_{1}}{h^{2}} \frac{\partial^{2} C_{1}}{\partial \eta^{2}}-K_{1} C_{1}
$$

Region-2

$$
\frac{1}{t} \frac{\partial C_{2}}{\partial \theta_{2}}+\frac{u_{2 x}}{L_{2}} \frac{\partial C_{2}}{\partial \xi_{2}}=\frac{D_{2}}{h^{2}} \frac{\partial^{2} C_{2}}{\partial \eta^{2}}-K_{2} C_{2}
$$

where $L_{1}$ and $L_{2}$ are the typical lengths along the flow direction. Following the Taylor (1953), we now assume that partial equilibrium is established in any cross-section of the channel so that the variations of $C_{1}$ and $C_{2}$ with $\eta$ are calculated from Eqs. (17) and (18) as

Region-1

Region-2

$$
\frac{\partial^{2} C_{1}}{\partial \eta^{2}}-\alpha_{1}^{2} C_{1}=\frac{h^{2}}{D_{1} L_{1}} u_{1 x} \frac{\partial C_{1}}{\partial \xi_{1}}
$$

$$
\frac{\partial^{2} C_{2}}{\partial \eta^{2}}-\alpha_{2}^{2} C_{2}=\frac{h^{2}}{D_{2} L_{2}} u_{2 x} \frac{\partial C_{2}}{\partial \xi_{2}}
$$

where $\alpha_{1}=h \sqrt{K_{1} / D_{1}}$ and $\alpha_{2}=h \sqrt{K_{2} / D_{2}}$

To solve these equations we use the following three types of boundary conditions:

The first one is connected with insulated type of boundary conditions, namely 


$$
\frac{\partial C_{1}}{\partial \eta}=0 \text { at } \eta=-1 \text { and } \frac{\partial C_{2}}{\partial \eta}=0 \text { at } \eta=1
$$

which expresses the fact that the walls of the channel are impermeable. However, in many biological problems the condition at the lower wall is insulating and the upper is conducting. In other words

$$
\frac{\partial C_{1}}{\partial \eta}=0 \quad \text { at } \eta=-1 \quad \text { and } \quad C_{2}=1 \text { at } \eta=1
$$

where the former represents the impermeable (insulating) and the later the permeable (conducting). The other condition can also be taken as the lower wall as conducting and upper wall is insulating

$$
C_{1}=1 \quad \text { at } \eta=-1 \quad \text { and } \quad \frac{\partial C_{2}}{\partial \eta}=0 \quad \text { at } \quad \eta=1
$$

where the former represents the permeable and the later the impermeable.

To find the exact solutions of Eqs. (19) and (20), we require two more interface conditions along with boundary conditions (21) to (23) for three different cases which are given by

$$
C_{1}=C_{2} \text { and } \frac{\partial C_{1}}{\partial \eta}=\frac{D_{2}}{D_{1}} \frac{\partial C_{2}}{\partial \eta} \text { at } \eta=0
$$

That is, the continuity of concentration and the continuity of mass flux at the interface.

Equations (19) and (20) are solved exactly for $C_{1}$ and $C_{2}$ which are given by

Region-1

$$
C_{1}=b_{1} \cosh \left(\alpha_{1} \eta\right)+b_{2} \sinh \left(\alpha_{1} \eta\right)+\frac{h^{2}}{D_{1} L} \frac{\partial C_{1}}{\partial \xi_{1}}\left(\frac{a_{1} \cosh \left(\sigma_{1} \eta\right)}{\left(\sigma_{1}^{2}-\alpha_{1}^{2}\right)}+\frac{a_{2} \sinh \left(\sigma_{1} \eta\right)}{\left(\sigma_{1}^{2}-\alpha_{1}^{2}\right)}-\frac{l_{1}}{\alpha_{1}^{2}}\right)
$$

Region-2

$$
C_{2}=b_{3} \cosh \left(\alpha_{2} \eta\right)+b_{4} \sinh \left(\alpha_{2} \eta\right)+\frac{h^{2}}{D_{2} L} \frac{\partial C_{2}}{\partial \xi_{2}}\left(\frac{a_{3} \cosh \left(\sigma_{2} \eta\right)}{\left(\sigma_{2}^{2}-\alpha_{2}^{2}\right)}+\frac{a_{4} \sinh \left(\sigma_{2} \eta\right)}{\left(\sigma_{2}^{2}-\alpha_{2}^{2}\right)}-\frac{l_{2}}{\alpha_{2}{ }^{2}}\right)
$$

where $b_{1}, b_{2}, b_{3}$ and $b_{4}$ are the integrating constants which are evaluated using boundary and interface conditions as defined in Eqs. (21) and (24). The expressions for $C_{1}$ and $C_{2}$ can also be written as

$$
C_{1}=\frac{h^{2}}{D_{1} L} \frac{\partial C_{1}}{\partial \xi_{1}} C_{11}+\frac{h^{2}}{D_{2} L} \frac{\partial C_{2}}{\partial \xi_{2}} C_{12} ; C_{2}=\frac{h^{2}}{D_{1} L} \frac{\partial C_{1}}{\partial \xi_{1}} C_{21}+\frac{h^{2}}{D_{2} L} \frac{\partial C_{2}}{\partial \xi_{2}} C_{22}
$$

where

$$
\begin{aligned}
& C_{11}=b_{11} \cosh \left(\alpha_{1} \eta\right)+b_{21} \sinh \left(\alpha_{1} \eta\right)+\frac{a_{1} \cosh \left(\sigma_{1} \eta\right)}{\sigma_{1}^{2}-\alpha_{1}^{2}}+\frac{a_{2} \sinh \left(\sigma_{1} \eta\right)}{\sigma_{1}^{2}-\alpha_{1}^{2}}-\frac{l_{1}}{\alpha_{1}^{2}}, \\
& C_{12}=b_{12} \cosh \left(\alpha_{1} \eta\right)+b_{22} \sinh \left(\alpha_{1} \eta\right), \\
& C_{21}=b_{31} \cosh \left(\alpha_{2} \eta\right)+b_{41} \sinh \left(\alpha_{2} \eta\right) \\
& C_{22}=b_{32} \cosh \left(\alpha_{2} \eta\right)+b_{42} \sinh \left(\alpha_{2} \eta\right)+\frac{a_{3} \cosh \left(\sigma_{2} \eta\right)}{\sigma_{2}^{2}-\alpha_{2}^{2}}+\frac{a_{4} \sinh \left(\sigma_{2} \eta\right)}{\sigma_{2}^{2}-\alpha_{2}^{2}}-\frac{l_{2}}{\alpha_{2}^{2}}
\end{aligned}
$$

The volumetric flow rates at which the solute is transported across a section of the channel of unit breadth $Q_{1}$ (region-1) and $Q_{2}$ (region-2) using Eqs. (14), (15) and (25), (26) are

Region-1

$$
Q_{1}=h \int_{-1}^{0} C_{1} u_{1 x} d \eta=-\left(Q_{11}+Q_{12}\right)
$$

where

Region-2

$$
Q_{11}=-\frac{h^{3}}{D_{1} L} \frac{\partial C_{1}}{\partial \xi_{1}} \int_{-1}^{0} C_{11} u_{1 x} d \eta \text { and } Q_{12}=-\frac{h^{3}}{D_{2} L} \frac{\partial C_{2}}{\partial \xi_{2}} \int_{-1}^{0} C_{12} u_{1 x} d \eta
$$

$$
Q_{2}=h \int_{0}^{1} C_{2} u_{2 x} d \eta=-\left(Q_{21}+Q_{22}\right)
$$

where

$$
Q_{21}=-\frac{h^{3}}{D_{1} L} \frac{\partial C_{1}}{\partial \xi_{1}} \int_{0}^{1} C_{21} u_{2 x} d \eta \text { and } Q_{22}=-\frac{h^{3}}{D_{2} L} \frac{\partial C_{2}}{\partial \xi_{2}} \int_{0}^{1} C_{22} u_{2 x} d \eta
$$


Following Taylor (1953), we assume that the variations of $C_{1}$ and $C_{2}$ with $\eta$ are small compared with those in the longitudinal direction, and if $C_{m 1}$ and $C_{m 2}$ are the mean concentration over a section, $\partial C_{1} / \partial \xi_{1}$ and $\partial C_{2} / \partial \xi_{2}$ are indistinguishable from $\partial C_{1} / \partial \xi_{1}$ and $\partial C_{2} / \partial \xi_{2}$ respectively so that Eqs. (27) and (28) may be written as

Region-1

Region-2

$$
Q_{11}=-D_{11}^{*} \frac{\partial C_{m 1}}{\partial \xi_{1}}, Q_{12}=-D_{12}^{*} \frac{\partial C_{m 2}}{\partial \xi_{2}}
$$

$$
Q_{21}=-D_{21}^{*} \frac{\partial C_{m 1}}{\partial \xi_{1}} \text { and } Q_{22}=-D_{22}^{*} \frac{\partial C_{m 2}}{\partial \xi_{2}}
$$

The fact that no material is lost in the process is expressed by the continuity equation for $C_{m 1}$ and $C_{m 2}$, namely

Region-1

$$
\frac{\partial Q_{11}}{\partial \xi_{1}}=-2 \frac{\partial C_{m 1}}{\partial t}, \frac{\partial Q_{12}}{\partial \xi_{2}}=-2 \frac{\partial C_{m 2}}{\partial t}
$$

Region-2

$$
\frac{\partial Q_{21}}{\partial \xi_{1}}=-2 \frac{\partial C_{m 1}}{\partial t}, \frac{\partial Q_{22}}{\partial \xi_{2}}=-2 \frac{\partial C_{m 2}}{\partial t}
$$

Equations (29) and (30) using eqs. (27) and (28) become

Region-1

$$
\frac{\partial C_{m 1}}{\partial t}=\frac{D_{11}^{*}}{2} \frac{\partial^{2} C_{m 1}}{\partial \xi_{1}^{2}}, \frac{\partial C_{m 2}}{\partial t}=\frac{D_{12}^{*}}{2} \frac{\partial^{2} C_{m 2}}{\partial \xi_{2}^{2}}
$$

Region-2

$$
\frac{\partial C_{m 1}}{\partial t}=\frac{D_{21}^{*}}{2} \frac{\partial^{2} C_{m 1}}{\partial \xi_{1}^{2}}, \frac{\partial C_{m 2}}{\partial t}=\frac{D_{22}^{*}}{2} \frac{\partial^{2} C_{m 2}}{\partial \xi_{2}^{2}}
$$

which are the equations governing the longitudinal dispersion,

where $D_{11}^{*}=\frac{h^{2}}{2 D_{1}} \int_{-1}^{0} C_{11} u_{1 x} d \eta=\frac{h^{2}}{2 D_{1}} F_{11}\left(\sigma_{1}, \sigma_{2}, p_{1}, p_{2}, \alpha_{1}, \alpha_{2}, m, n\right)$,

$$
\begin{aligned}
& D_{12}^{*}=\frac{h^{2}}{2 D_{2}} \int_{-1}^{0} C_{12} u_{1 x} d \eta=\frac{h^{2}}{2 D_{2}} F_{12}\left(\sigma_{1}, \sigma_{2}, p_{1}, p_{2}, \alpha_{1}, \alpha_{2}, m, n\right), \\
& D_{21}^{*}=\frac{h^{2}}{2 D_{1}} \int_{0}^{1} C_{21} u_{2 x} d \eta=\frac{h^{2}}{2 D_{1}} F_{21}\left(\sigma_{1}, \sigma_{2}, p_{1}, p_{2}, \alpha_{1}, \alpha_{2}, m, n\right), \\
& D_{22}^{*}=\frac{h^{2}}{2 D_{2}} \int_{0}^{1} C_{22} u_{2 x} d \eta=\frac{h^{2}}{2 D_{2}} F_{22}\left(\sigma_{1}, \sigma_{2}, p_{1}, p_{2}, \alpha_{1}, \alpha_{2}, m, n\right) .
\end{aligned}
$$

For lower wall impermeable and upper wall permeable, the solutions remain the same as in Eqs. (25) and (26), whereas the integrating constants are evaluated as defined in Eqs. (22) and (24). The integrating constants of Eqs. (25) and (26) are evaluated using boundary and interface conditions as defined in Eqs. (23) and (24) foe lower wall permeable and upper wall impermeable wall conditions. The evaluation of effective dispersion coefficient $F_{i i}$ and volumetric flow rate for lower wall impermeable and upper wall permeable and lower wall permeable and upper wall impermeable remain the same as defined above for imperameable wall conditions.

$F_{i i}$ is used in terms of $F_{1}=F_{11}+F_{12}$ for region-1 and $F_{2}=F_{21}+F_{22}$ for region-2 for computations. Values of $F_{i i}$ are computed for different values of dimensionless parameters such as viscosity ratio $m$, pressure gradients $p_{1}, p_{2}$ and porous parameters $\sigma_{1}, \sigma_{2}$ for variations of $\alpha_{1}$ and $\alpha_{2}$ for three different wall conditions and are shown in Figures 2, 4 and 6. Volumetric flow rate is also computed for variations of viscosity ratio, pressure gradients and porous parameters and displayed in Figures 3 and 5.

\section{Case 2 Diffusion with combined homogeneous and heterogeneous first-order chemical reaction}

We now discuss the problem of diffusion in a channel with a first-order irreversible chemical reaction taking place both in the bulk of the fluid as well as at the walls which are assumed to be catalytic. In this case the diffusion equations remain the same as defined in Eqs. (19) and (20) subject to the dimensionless boundary and interface conditions as 


$$
\begin{gathered}
\frac{\partial C_{1}}{\partial \eta}-\beta_{1} C_{1}=0 \text { at } \eta=-1 \\
\frac{\partial C_{2}}{\partial \eta}+\beta_{2} C_{2}=0 \text { at } \eta=1 \\
C_{1}=C_{2} \text { at } \eta=0 \\
D_{1} \frac{\partial C_{1}}{\partial \eta}=D_{2} \frac{\partial C_{2}}{\partial \eta} \text { at } \eta=0
\end{gathered}
$$

where $\beta_{1}=f_{1} h$ and $\beta_{2}=f_{2} h$ are the heterogeneous reaction rate parameters corresponding to catalytic reaction at the walls. The solutions remain the same as defined in Eqs. (25) and (26) but the integrating constants $b_{1}, b_{2}, b_{3}$ and $b_{4}$ are obtained using boundary and interface conditions as defined in Eq. (33). The effective dispersion coefficient $F_{i i}$ is shown in Figure 7.

\section{Case 3: The channel filled with porous matrix (one fluid model)}

To validate the results obtained for composite porous medium, (two fluid model) the problem is solved considering the channel filled with only porous medium (one fluid model) which was studied by Rudraiah and $\mathrm{Ng}$ (2007). The solutions for one fluid model for impermeable wall conditions obtained by Rudraiah and Ng (2007) is given in Case 2 of Prathap Kumar et al. (2011). The solutions for one fluid model with lower impermeable and upper permeable wall condition, with lower permeable and upper impermeable wall conditions and heterogeneous wall conditions are evaluated. The values for the effective diffusion coefficient $F(\alpha, \sigma, p)$ and mass flow rate are computed for all the four different wall boundary conditions and are shown in Table 2(a, b) for two fluid (present model) and one fluid model (Rudraiah and Ng, 2007).

\section{Case 4: The channel filled with purely viscous fluid}

Since the results for composite porous medium (two fluid model) tally with Rudraiah and $\mathrm{Ng}$ (2007) (one fluid model) for all values of porous parameter $\sigma$, we further justify our results by comparing with the results obtained by Gupta and Gupta (1972) for one fluid model in the absence of porous parameter. The solutions of Eqs. (5) and (6) using boundary and interface conditions (7) in the absence of porous parameter $\sigma$ remain the same as given in Case 3a of Prathap Kumar et al. (2011). The solutions of one fluid model (the channel filled with only viscous fluid) obtained by Gupta and Gupta (1972) is given in case 3b of Prathap Kumar et al. (2011) and hence not shown here. The solution for heterogeneous chemical reaction is also found for two fluid and one fluid model in the absence of porous matrix and the results are shown in Table 3(a,b). All the constants appeared above are defined in the appendix.

\section{Results and discussion}

The problem concerned is with the longitudinal dispersion of a solute subject to molecular diffusion when it is introduced into a channel for composite porous medium following Taylor's dispersion model with a homogeneous and heterogeneous first-order chemical reaction. In order to find out average velocity in both the regions, no-slip conditions at the boundaries and continuity of velocity and shear stress is assumed at the interface. The volumetric flow rate in both the regions of the channel is found. The effective dispersion coefficient in the each region is also evaluated and the results are shown graphically for variations of governing parameters. The homogeneous first-order chemical reaction is analyzed for three types of boundary conditions. The first one is connected with impermeable type of boundary conditions, second one is that lower wall impermeable and the upper wall permeable and third one is that the lower wall permeable and upper wall impermeable. The effective dispersion coefficient is also found with a heterogeneous first-order chemical reaction. The physical parameters such as viscosity ratio, pressure gradient

$p\left(=p_{1}=p_{2}\right)$ and porous parameter $\sigma\left(=\sigma_{1}=\sigma_{2}\right)$ are fixed as 1,1 and 4 respectively except the varying parameters in all the graphs.

\section{Case 1: Diffusion with a homogeneous first-order chemical reaction}

Concentration distribution with impermeable wall conditions

The effective dispersion coefficient $F\left(=F_{1}\left(\alpha_{1}, \alpha_{2}\right)\right.$ of region- $1+F_{2}\left(\alpha_{1}, \alpha_{2}\right)$ of region-2) for variations of viscosity ratio $m$ (Figure 2a), pressure gradient (Figure 2b) and porous parameter (Figure 2c) is shown in Figure 2. As the reaction rate parameter $\alpha\left(=\alpha_{1}=\alpha_{2}\right)$ increases $F$ decreases for any value of viscosity ratio $m$, pressure gradient $p$ and porous parameter $\sigma$.

As the viscosity ratio $m$ increases, the total effective dispersion coefficient increases. It is observed from Figure $2 b$ that as pressure gradient $p$ increases the effective dispersion coefficient $F$ increases. It is also seen from Figure $2 \mathrm{~b}$ that for small value of $p=0.1$ there is zero value of effective dispersion coefficient. This is due to the fact that for small value of pressure gradient 
there will be no flow and hence there will be no dispersion. It is also observed that the effective dispersion coefficient remains the same irrespective of the direction of the pressure gradient. It is observed that the effective dispersion coefficient $F$ decreases very rapidly with increasing porous parameter $\sigma$ which is the similar result obtained by Rudraiah and $\mathrm{Ng}$ (2007). This is due to the fact that as $\sigma$ grows, the velocity profiles continue to flatten out and tend to plug flow as $\sigma \rightarrow \infty$. This flattening effect is the cause for the decrease in $F$ with an increase in $\sigma$.
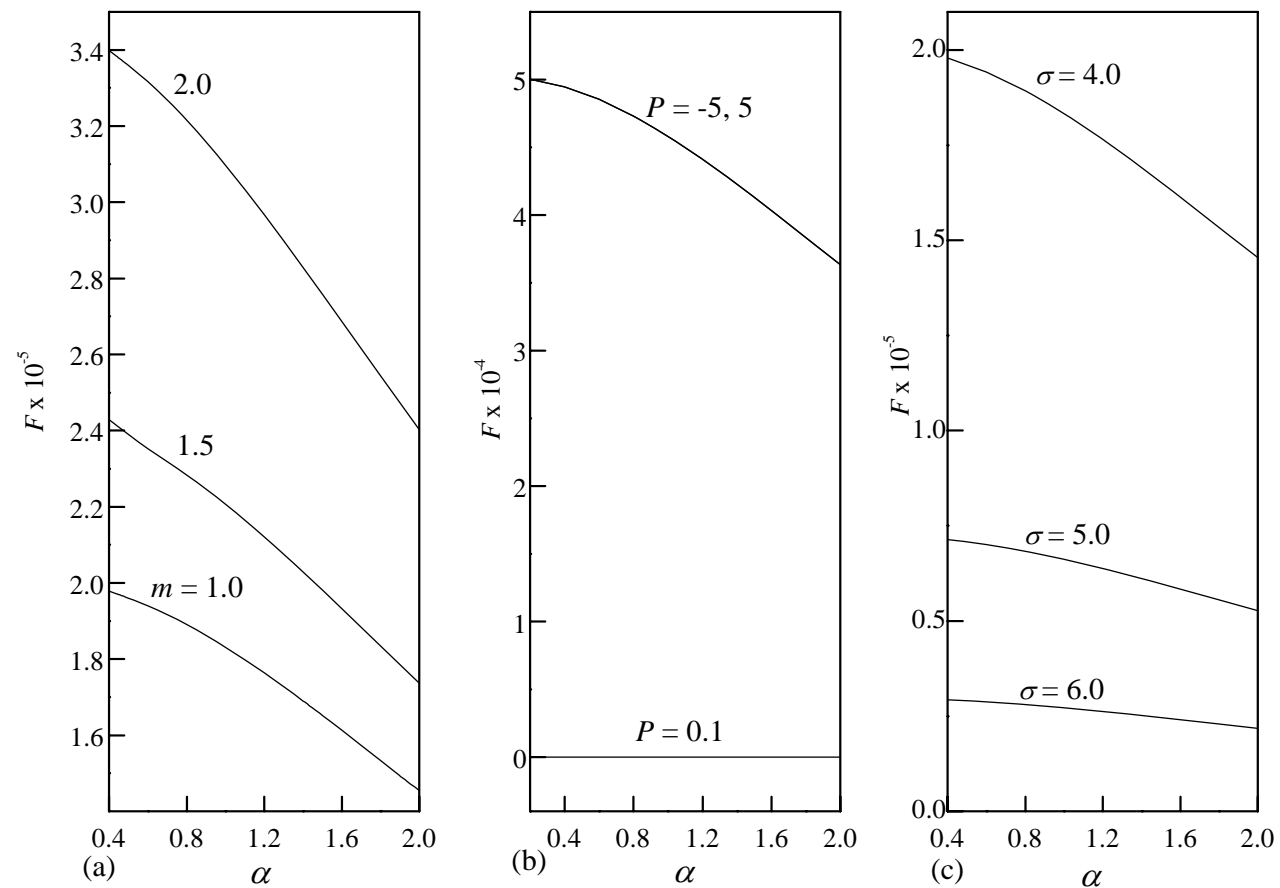

Figure 2: Effective dispersion coefficient $F$ versus $\alpha$ for different values of a) viscosity ratio $m$, b) pressure gradient $p$ and c) porous parameter $\sigma$ for impermeable wall condition.
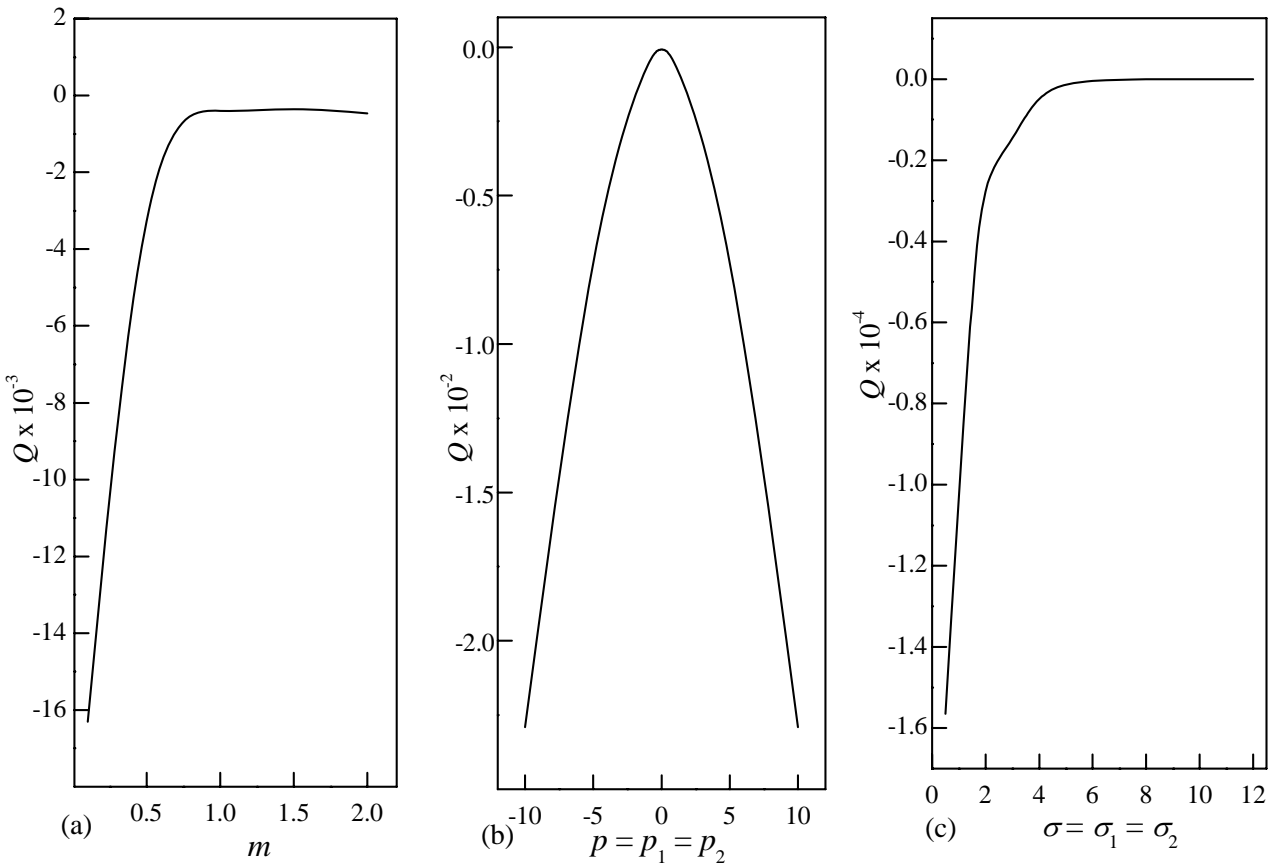

Figure 3: Volumetric flow rate versus a) viscosity ratio, b) pressure gradient and c) porous parameter for impermeable wall conditions. 
The effects of viscosity ratio, pressure gradient and porous parameter on volumetric flow rate are shown in Figure 3 . It is seen that as the viscosity ratio $m$ and porous parameter $\sigma\left(=\sigma_{1}=\sigma_{2}\right)$ increases, volumetric flow rate increases for small values of $m$ (Figure 3a), $\sigma$ (Figure 3c) and remains almost constant for values of viscosity ratio $m$ greater than 0.8 and porous parameter $\sigma$ greater than 5 . Volumetric flow rate is symmetric for negative and positive values of pressure gradient and the optimum flow rate is attained in the absence of pressure gradient (Figure 3b).

\section{Concentration distribution with lower wall impermeable and upper wall permeable wall conditions}

The effects of viscosity ratio $m$, porous parameter $\sigma\left(=\sigma_{1}=\sigma_{2}\right)$ and pressure gradient $p$ on the effective dispersion coefficient with variation of homogeneous chemical reaction rate parameter $\alpha\left(=\alpha_{1}=\alpha_{2}\right)$ is shown in Figure 4. As the reaction rate parameter $\alpha$ increases, the effective dispersion coefficient decreases for any values of $m$ (Figure $4 \mathrm{a}$ ) and $\sigma$ (Figure 4c). For positive value of pressure gradient $(p=5)$ the effective dispersion coefficient $F$ decreases as the chemical reaction rate parameter $\alpha$ increases. Reversal effect is observed for negative value of $p(p=-5)$. For small value of pressure gradient $p=0.1$, there is no effect of $\alpha$ on $F$.

As the viscosity ratio $m$ increases the effective dispersion coefficient $F$ decreases as seen in Figure 4a. As the pressure gradient increases the effective dispersion coefficient $F$ decreases. As the porous parameter $\sigma$ increases effective dispersion coefficient $F$ increases as seen in seen in Figure 4c.

The effect of viscosity ratio $m$ (Figure 5a), porous parameter $\sigma$ (Figure $5 c$ ) and pressure gradient $p$ (Figure $5 b$ ) on the volumetric flow rate is depicted in Figure 5. It is seen that as the viscosity ratio increases volumetric flow rate increases for small values of $m$ and remains almost constant for values of viscosity ratio $m$ greater than 1.0. Volumetric flow rate is symmetric for negative and positive values of pressure gradients. Volumetric flow rate decreases as porous parameter increases and remains almost constant for values of porous parameter $\sigma\left(=\sigma_{1}=\sigma_{2}\right)$ greater than 15 .

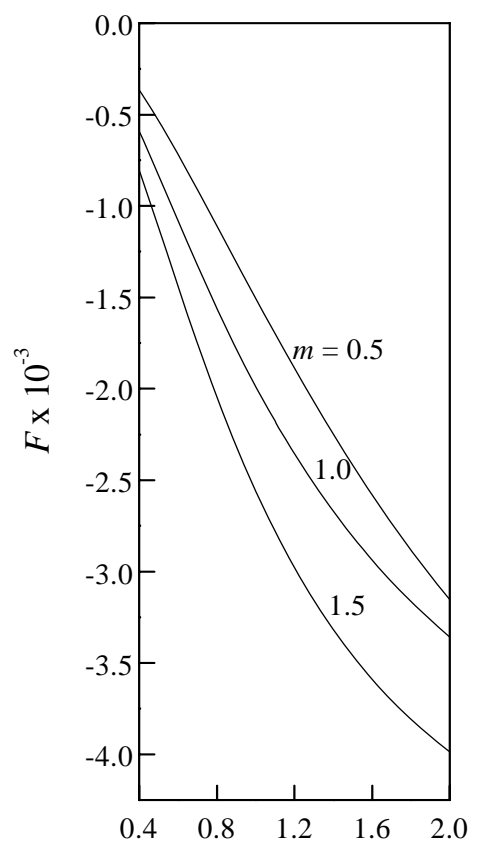

(a)
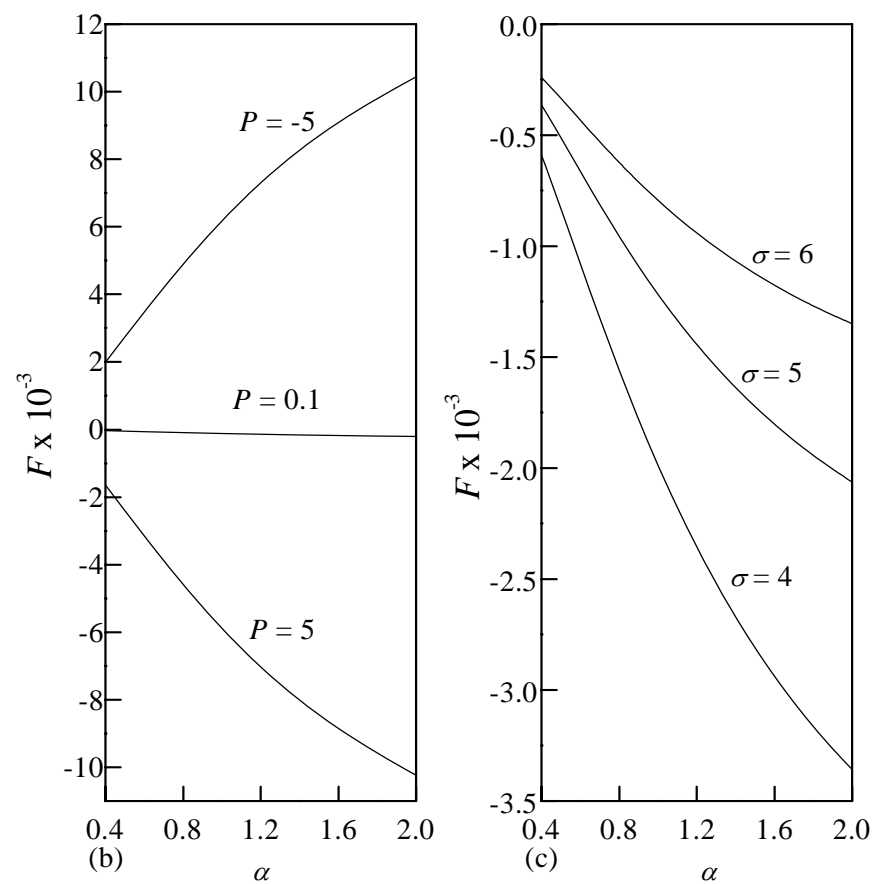

Figure 4: Effective dispersion coefficient $F$ versus $\alpha$ for different values of a) viscosity ratio $m$,

b) pressure gradient $p$ and c) porous parameter $\sigma$ for impermeable and permeable wall conditions. 

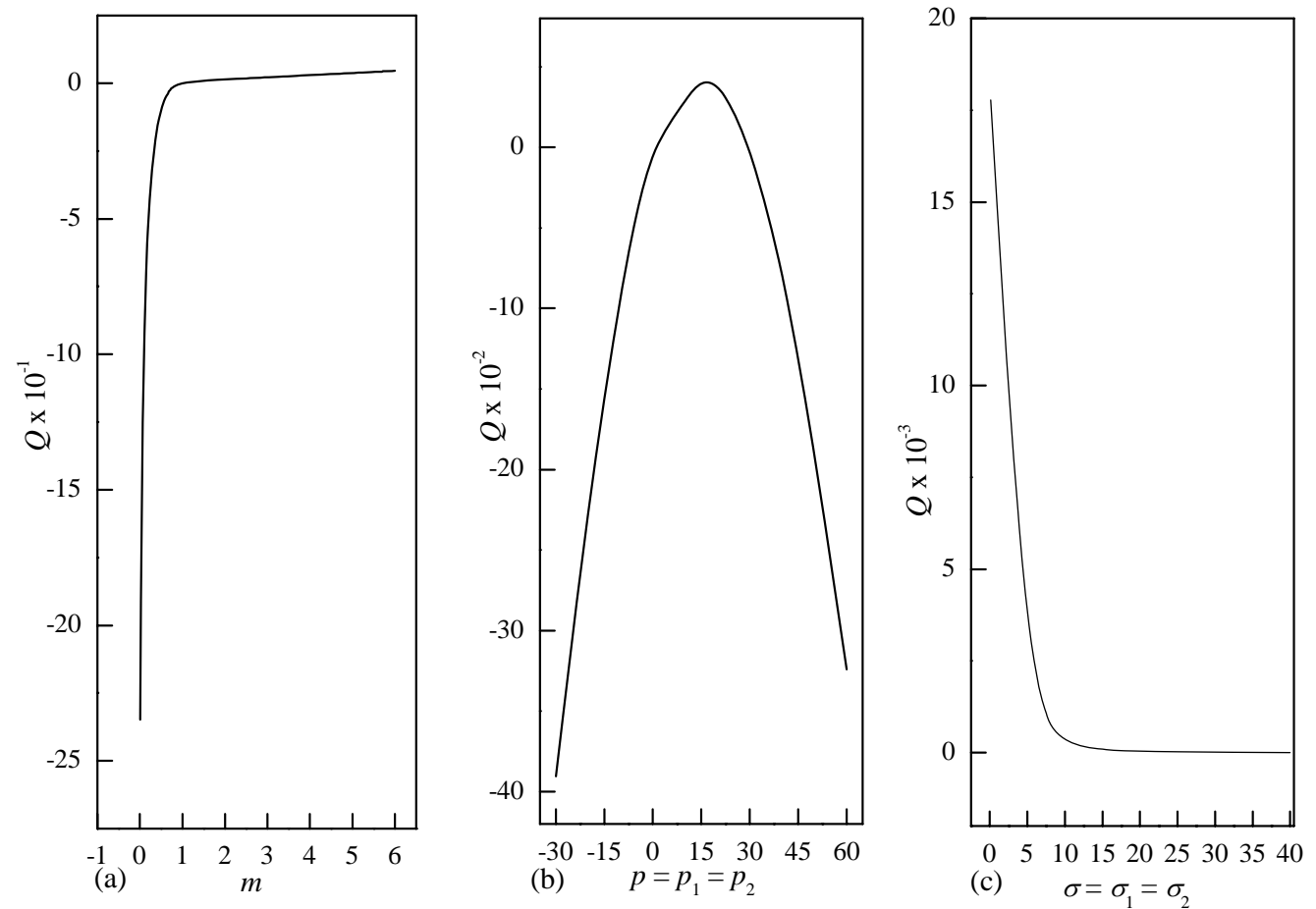

Figure 5. Volumetric flow rate versus a) viscosity ratio, b) pressure gradient and

c) porous parameter for lower wall impermeable and upper wall permeable wall conditions.
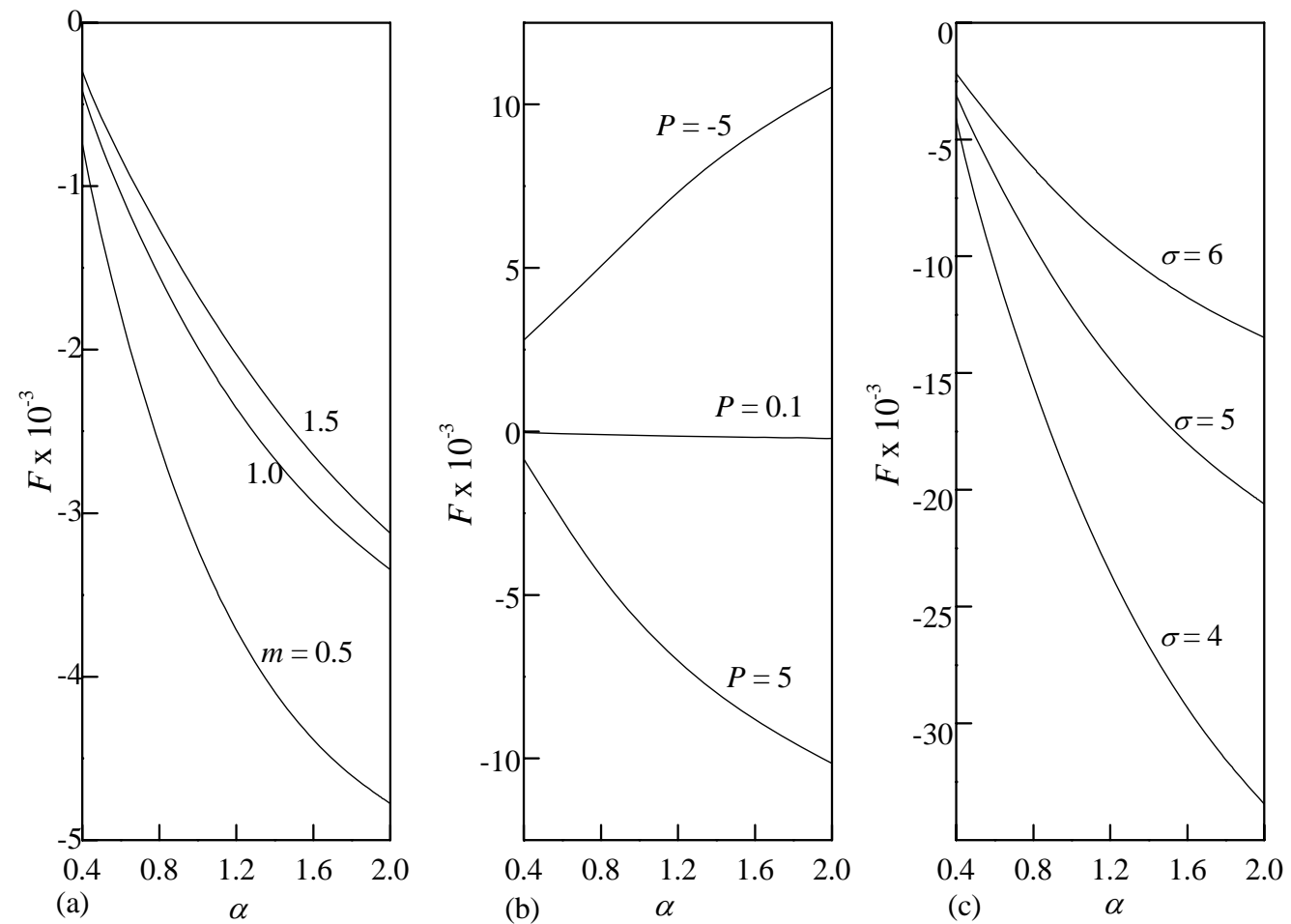

Figure 6: Effective dispersion coefficient $F$ versus $\alpha$ for different values of a) viscosity ratio $m$,

b) pressure gradient $p$ and c) porous parameter $\sigma$ for permeable and impermeable wall conditions. 
Concentration distribution with lower wall permeable and upper wall impermeable wall conditions.

The effects of viscosity ratio $m$, porous parameter and pressure gradient $p$ on the effective dispersion coefficient with variation of homogeneous chemical reaction rate parameter $\alpha$ is shown in Figure 6. As the reaction rate parameter $\alpha$ increases, the effective dispersion coefficient decreases for any values of $m$ (Figure 6a) and $\sigma$ (Figure 6c). For values of $p<1$, the total effective dispersion coefficient $F$ increases, where as it decreases for values of $p>1$ (Figure 6b) as $\alpha$ increases. As the viscosity ratio $m$ increases, the effective dispersion coefficient $F$ increases (Figure 6a). As pressure gradient $p$ increases $F$ decreases as seen in Figure 6b. As the porous parameter $\sigma$ increases the effective dispersion coefficient $F$ increases (Figure 6c). The effects of $p$ and $\sigma$ on $F$ is the similar result observed for impermeable and permeable wall conditions.

The effect of viscosity ratio $m$, porous parameter $\sigma\left(=\sigma_{1}=\sigma_{2}\right)$ and pressure gradients $p$ on the volumetric flow rate shows the similar effect as that of impermeable and permeable wall conditions (Figure 5). That is, as the viscosity ratio increases volumetric flow rate increases for small values of $m$, remains almost constant for values of viscosity ratio $m$ greater than 1.0. Volumetric flow rate is symmetric for negative and positive values of pressure gradients. Volumetric flow rate decreases as porous parameter increases and remains almost constant for increasing values of porous parameter $\sigma$.
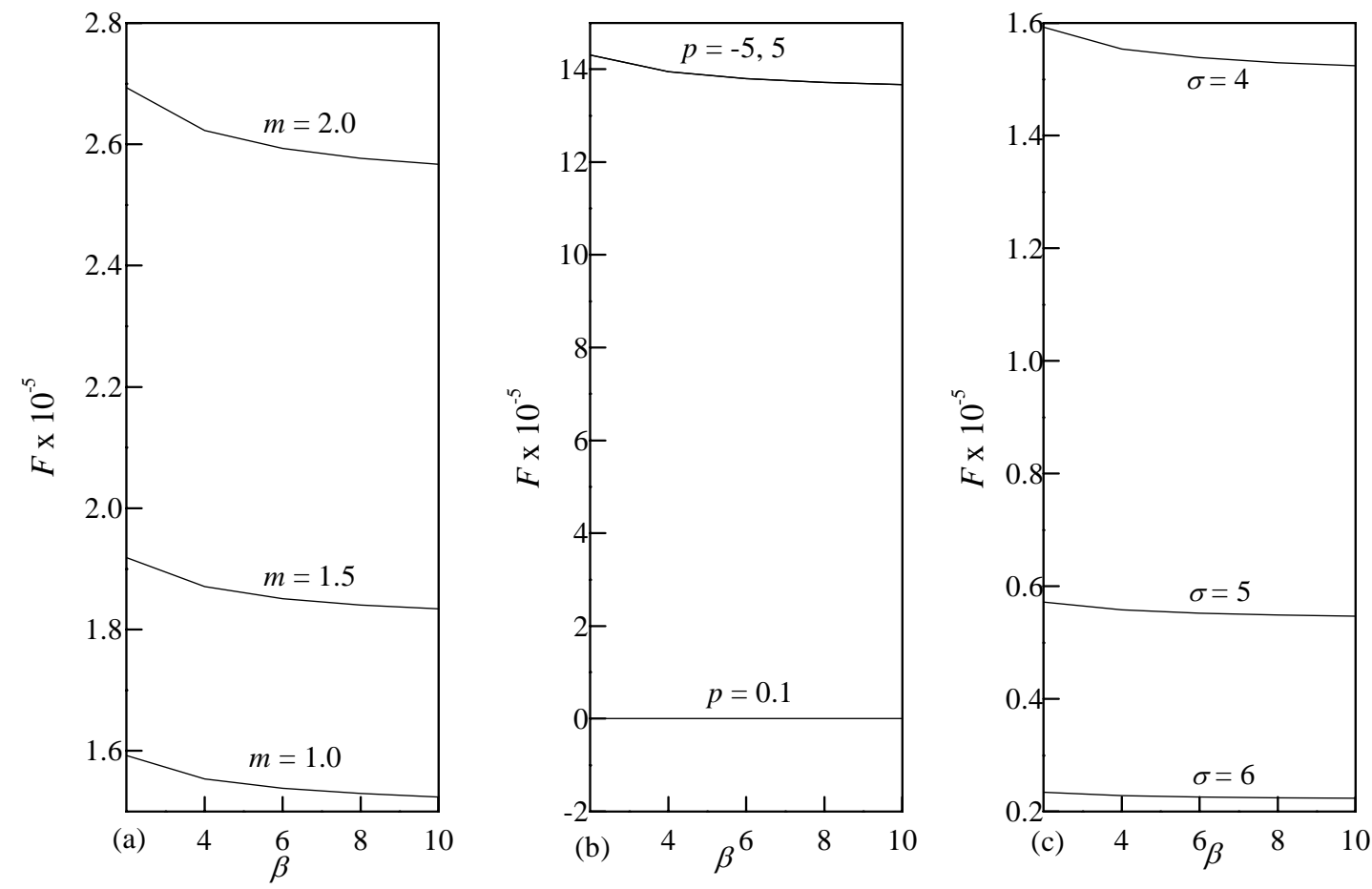

Figure 7: Effective dispersion coefficient $F$ versus $\beta$ for different values of a) viscosity ratio $m$, b) pressure gradient $p$ and c) porous parameter $\sigma$ for combined homogeneous and heterogeneous impermeable wall conditions.

\section{Case 2: Diffusion with combined homogeneous and heterogeneous first-order chemical reaction}

The effect of heterogeneous reaction rate parameter $\beta$ for a fixed value of homogeneous reaction rate parameter $\alpha=1$ is shown in Figure 7. From this it is clear that the increase in the wall catalytic parameter causes decrease in the effective dispersion coefficient for all values of viscosity ratio $m$ (Figure 7a), pressure gradient $p$ (Figure 7b) and porous parameter $\sigma$ (Figure 7c). As the viscosity ratio $m$ increases, the effective dispersion coefficient $F$ increases as seen in Figure 7a. The effect of pressure gradient $p$ (Figure $7 \mathrm{~b}$ ) on $F$ is similar to impermeable wall condition (Figure 3b). The effective dispersion coefficient $F$ decreases very rapidly as porous parameter $\sigma$ increases (Figure 7c) which is the similar result as in the case of homogeneous reaction. This result is similar to the result obtained by Rudraiah and $\mathrm{Ng}$ (2007) for heterogeneous reactions.

The effect of viscosity ratio, pressure gradient and porous parameter on volumetric flow rate shows the similar result as that of homogeneous chemical reaction with impermeable wall conditions (Figure 3). 
To understand the nature of distribution of concentration for the real field, physical numbers for porous parameter and diffusivity coefficients are chosen and shown in Table 1. It is assumed that $\frac{\partial C_{1}}{\partial \xi_{1}}=\frac{\partial C_{2}}{\partial \xi_{2}}=1$ for evaluating the values of concentration in Table 1 for four wall boundary conditions. The experimental values of diffusion coefficients in gases at one atmosphere are chosen from Cussler (1998). It is interesting to note that for any combination of gases, as the porous parameter $\sigma$ increases, concentration decreases in magnitude in both the regions. This is due to the fact that an increase in the grain size increases the permeability $\kappa$ and hence porous parameter $\sigma$ decreases, which in turn reduces the value of the diffusion coefficient (this behavior is in conformity with the experimental results of Harlemann et. al., 1963) which results in the reduction of concentration. Two different fluids are chosen in each region such as Carbon dioxide and hydrogen, both the regions filled with air. Another combination of Nitrogen and Helium are chosen with both regions filled with water. It is seen that the concentration is high in magnitude for the channel filled with air compared to the channel filled with water in both the regions for variations of diffusivity coefficient for impermeable wall conditions.

For lower impermeable and upper wall permeable, the effect of $\sigma$ on the concentration is not effective when compared to impermeable wall conditions. The values of concentration are less for water when compared to air which is in contradiction with the result obtained for impermeable wall conditions as seen in Table 1.

For lower impermeable and upper wall impermeable, the effect of porous parameter $\sigma\left(=\sigma_{1}=\sigma_{2}\right)$ on the concentration is not effective when compared to impermeable wall conditions. The values of concentration are less for air when compared to water which is in contradiction with the result obtained for impermeable wall conditions.

For combined homogeneous and heterogeneous wall conditions, the values of concentration are less for water when compared to air which is the similar result obtained for homogeneous wall conditions.

Table 1. Values of concentration for homogeneous impermeable wall conditions.

\begin{tabular}{|c|c|c|c|c|c|c|}
\hline \multirow[b]{2}{*}{$\eta$} & \multicolumn{3}{|c|}{$D_{1}=0.148\left(\right.$ Air- $\left.\mathrm{CO}_{2}\right), D_{2}=0.710\left(\right.$ Air- $\left.\mathrm{H}_{2}\right)$} & \multicolumn{3}{|c|}{$D_{1}=0.293\left(\mathrm{~N}_{2}-\mathrm{H}_{2} \mathrm{O}\right), D_{2}=0.908\left(\mathrm{He}-\mathrm{H}_{2} \mathrm{O}\right)$} \\
\hline & $\begin{array}{c}\sigma=0.54 \\
\quad(\text { Soil) }\end{array}$ & $\begin{array}{c}\sigma=0.65 \\
\text { (Silica } \\
\text { grains) }\end{array}$ & $\begin{array}{c}\sigma=0.76 \\
\text { (wire cramps) }\end{array}$ & $\begin{array}{c}\sigma=0.54 \\
\quad \text { (Soil) }\end{array}$ & $\begin{array}{c}\sigma=0.65 \\
\text { (Silica } \\
\text { grains) }\end{array}$ & $\begin{array}{c}\sigma=0.76 \\
\text { (wire cramps) }\end{array}$ \\
\hline & \multicolumn{6}{|c|}{ impermeable wall conditons } \\
\hline-1.0 & -0.08326 & -0.07922 & -0.07489 & -0.039592 & -0.037677 & -0.035621 \\
\hline-0.6 & -0.03784 & -0.03595 & -0.03391 & -0.016455 & -0.015628 & -0.014742 \\
\hline-0.2 & 0.01402 & 0.01334 & 0.01261 & 0.010374 & 0.009868 & 0.009324 \\
\hline 0.2 & 0.01537 & 0.01462 & 0.0138 & 0.010960 & 0.010420 & 0.009841 \\
\hline 0.6 & 0.00218 & 0.00207 & 0.00196 & 8.4335E-4 & 8.0583E-4 & 7.6554E-4 \\
\hline \multirow[t]{2}{*}{1.0} & -0.00804 & -0.00766 & -0.00726 & -0.007084 & -0.006747 & -0.006386 \\
\hline & \multicolumn{6}{|c|}{ lower wall impermeable and upper wall permeable } \\
\hline-1.0 & 0.294432 & 0.298323 & 0.302497 & 0.316676 & 0.318472 & 0.320400 \\
\hline-0.6 & 0.370463 & 0.372206 & 0.374073 & 0.368697 & 0.369394 & 0.370142 \\
\hline-0.2 & 0.519153 & 0.518281 & 0.517346 & 0.486860 & 0.486194 & 0.485479 \\
\hline 0.2 & 0.628501 & 0.627512 & 0.626451 & 0.598943 & 0.598207 & 0.597417 \\
\hline 0.6 & 0.751974 & 0.751588 & 0.751175 & 0.738568 & 0.738284 & 0.737979 \\
\hline 1.0 & 1.000000 & 1.000000 & 1.000000 & 1.000000 & 1.000000 & 1.000000 \\
\hline & \multicolumn{6}{|c|}{ lower wall permeable and upper wall impermeable } \\
\hline-1.0 & 1.0000000 & 1.0000000 & 1.0000000 & 1.000000 & 1.000000 & 1.000000 \\
\hline-0.6 & 0.6138659 & 0.6133354 & 0.6127653 & 0.630908 & 0.630541 & 0.630147 \\
\hline-0.2 & 0.3398566 & 0.3379621 & 0.3359297 & 0.370473 & 0.369302 & 0.368047 \\
\hline 0.2 & 0.1762324 & 0.1748759 & 0.1734208 & 0.219693 & \begin{tabular}{|l}
0.218768 \\
\end{tabular} & 0.217776 \\
\hline 0.6 & 0.1322007 & 0.1316143 & 0.1309851 & 0.169566 & 0.169217 & 0.168843 \\
\hline 1.0 & 0.1122290 & 0.1121617 & 0.1120897 & 0.148985 & 0.149034 & 0.149087 \\
\hline & \multicolumn{6}{|c|}{ combined homogeneous and heterogeneous wall conditions } \\
\hline-1.0 & -0.044417 & -0.042264 & -0.039954 & -0.020443 & -0.019453 & -0.018391 \\
\hline-0.6 & -0.014101 & -0.013354 & -0.012553 & -0.004150 & -0.003918 & -0.003670 \\
\hline-0.2 & 0.026517 & 0.025233 & 0.023855 & 0.017829 & \begin{tabular}{|l|}
0.016963 \\
\end{tabular} & 0.016034 \\
\hline 0.2 & 0.022301 & 0.021211 & 0.020042 & 0.016007 & 0.015225 & 0.014386 \\
\hline 0.6 & 0.008304 & 0.007909 & 0.007484 & 0.005534 & 0.005272 & 0.004991 \\
\hline 1.0 & -0.001722 & -0.001643 & -0.001558 & -0.001989 & -0.001896 & -0.001796 \\
\hline
\end{tabular}




\section{Case 3: The channel filled with porous matrix (one fluid model)}

The problem of dispersion in porous media with and without chemical reaction was analysed by Rudraiah and Ng (2007) in which the channel is filled with only one porous matrix.

The Table 2(a, b) display the values of effective diffusion coefficient and volumetric flow rate for the two fluid (present model) and one-fluid (Rudraiah and Ng, 2007) model for homogeneous impermeable, lower wall impermeable and upper wall permeable, lower wall permeable and upper wall impermeable, and combined homogeneous and heterogeneous wall conditions. It is observed that for all the cases the effective dispersion coefficient $F$ and volumetric flow rate $Q$ agree very well with Rudraiah and $\mathrm{Ng}$ (2007) model which strongly justify our results in the presence of porous matrix.

\section{Case 4: The channel filled with only viscous fluid (Two fluid model) for homogeneous impermeable wall conditions}

In the absence of porous matrix, two fluid model agree very well with the results of Gupta and Gupta (1972) for one fluid model which further justify our model which is evident in Table 3(a,b) for effective dispersion coefficient and volumetric flow rate respectively.

Table 2a. Values of effective diffusion coefficient in the presence of porous matrix.

\begin{tabular}{|c|c|c|c|c|}
\hline & \multicolumn{3}{|c|}{ Two fluid model (present model) } & $\begin{array}{c}\text { One fluid model } \\
\text { Rudraiah and Ng }(2007)\end{array}$ \\
\hline & $F_{1}\left(\alpha_{1}, \alpha_{2}\right)$ & $F_{2}\left(\alpha_{1}, \alpha_{2}\right)$ & $F\left(\alpha_{1}, \alpha_{2}\right)$ & $F(\alpha)$ \\
\hline$\alpha$ & \multicolumn{4}{|c|}{ homogeneous impermeable wall conditions } \\
\hline 0.4 & $3.56801 \mathrm{E}-6$ & $3.56801 \mathrm{E}-6$ & $7.13602 \mathrm{E}-6$ & $7.13602 \mathrm{E}-6$ \\
\hline 0.8 & $3.41511 \mathrm{E}-6$ & $3.41511 \mathrm{E}-6$ & $6.83022 \mathrm{E}-6$ & $6.83022 \mathrm{E}-6$ \\
\hline 1.2 & $3.18869 \mathrm{E}-6$ & $3.18869 \mathrm{E}-6$ & $6.37737 \mathrm{E}-6$ & $6.37737 \mathrm{E}-6$ \\
\hline 1.6 & $2.91988 \mathrm{E}-6$ & $2.91988 \mathrm{E}-6$ & $5.83976 \mathrm{E}-6$ & $5.83976 \mathrm{E}-6$ \\
\hline 2.0 & $2.63695 \mathrm{E}-6$ & $2.63695 \mathrm{E}-6$ & $5.2739 \mathrm{E}-6$ & $5.2739 \mathrm{E}-6$ \\
\hline$\alpha$ & \multicolumn{5}{|c|}{ lower wall impermeable and upper wall permeable } \\
\hline 0.4 & $1.28569 \mathrm{E}-4$ & $-4.84811 \mathrm{E}-4$ & $3.5624 \mathrm{E}-4$ & $3.5624 \mathrm{E}-4$ \\
\hline 0.8 & $2.69952 \mathrm{E}-4$ & -0.0012317 & $9.6174 \mathrm{E}-4$ & $9.6174 \mathrm{E}-4$ \\
\hline 1.2 & $2.94069 \mathrm{E}-4$ & -0.0017429 & -0.0014489 & -0.0014489 \\
\hline 1.6 & $2.51804 \mathrm{E}-4$ & -0.0020586 & -0.0018068 & -0.0018068 \\
\hline 2.0 & $1.92032 \mathrm{E}-4$ & -0.0022556 & -0.0020635 & -0.0020635 \\
\hline$\alpha$ & \multicolumn{2}{|c|}{ lower wall permeable and upper wall impermeable } \\
\hline 0.4 & $1.28569 \mathrm{E}-4$ & $-4.84811 \mathrm{E}-4$ & $-3.5624 \mathrm{E}-4$ & $-3.5624 \mathrm{E}-4$ \\
\hline 0.8 & $2.69952 \mathrm{E}-4$ & -0.0012317 & $-9.6174 \mathrm{E}-4$ & $-9.6174 \mathrm{E}-4$ \\
\hline 1.2 & $2.94069 \mathrm{E}-4$ & -0.0017429 & -0.0014489 & -0.0014489 \\
\hline 1.6 & $2.51804 \mathrm{E}-4$ & -0.0020586 & -0.0018068 & -0.0018068 \\
\hline 2.0 & $1.92032 \mathrm{E}-4$ & -0.0022556 & -0.0020635 & -0.0020635 \\
\hline$\beta$ & \multicolumn{3}{|c|}{ combined homogeneous and heterogeneous wall conditions } \\
\hline 2 & $2.86083 \mathrm{E}-6$ & $2.86083 \mathrm{E}-6$ & $5.72166 \mathrm{E}-6$ & $5.72166 \mathrm{E}-6$ \\
\hline 4 & $2.78912 \mathrm{E}-6$ & $2.78912 \mathrm{E}-6$ & $5.57824 \mathrm{E}-6$ & $5.57824 \mathrm{E}-6$ \\
\hline 6 & $2.75983 \mathrm{E}-6$ & $2.75983 \mathrm{E}-6$ & $5.51967 \mathrm{E}-6$ & $5.51967 \mathrm{E}-6$ \\
\hline 8 & $2.74392 \mathrm{E}-6$ & $2.74392 \mathrm{E}-6$ & $5.48783 \mathrm{E}-6$ & $5.48783 \mathrm{E}-6$ \\
\hline 10 & $2.73392 \mathrm{E}-6$ & $2.73392 \mathrm{E}-6$ & $5.46783 \mathrm{E}-6$ & $5.46783 \mathrm{E}-6$ \\
\hline
\end{tabular}

Table $2 \mathbf{b}$. Values of volumetric flow rate in the presence of porous matrix.

\begin{tabular}{|c|c|c|c|}
\hline \multicolumn{3}{|c|}{ Two fluid model (present model) } & $\begin{array}{c}\text { One fluid model } \\
\text { Rudraiah and Ng (2007) }\end{array}$ \\
\hline$Q_{1}$ & $Q_{2}$ & $Q$ & $Q$ \\
\hline \multicolumn{4}{|c|}{ homogeneous impermeable wall conditions } \\
\hline$-3.30916 \mathrm{E}-6$ & $-3.30916 \mathrm{E}-6$ & $-6.61833 \mathrm{E}-6$ & $-6.61833 \mathrm{E}-6$ \\
\hline \multicolumn{3}{|c|}{ lower wall impermeable and upper wall permeable } \\
\hline$-2.94435 \mathrm{E}-4$ & 0.00151776 & 0.00122333 \\
\hline \multicolumn{4}{|c|}{ lower wall permeable and upper wall impermeable } \\
\hline$-2.94435 \mathrm{E}-4$ & 0.00151776 & 0.00122333 & 0.00122333 \\
\hline \multicolumn{3}{|c|}{ combined homogeneous and heterogeneous wall conditions } \\
\hline -2.95774E-6 & -2.95774E-6 & -5.91549E-6 & $-5.91549 \mathrm{E}-6$ \\
\hline
\end{tabular}


Table 3a. Values of effective diffusion coefficient for pure viscous fluid.

\begin{tabular}{|c|c|c|c|c|}
\hline & \multicolumn{3}{|c|}{ Two fluid model (present model) } & $\begin{array}{c}\text { One fluid model } \\
\text { Gupta and Gupta (1972) }\end{array}$ \\
\hline & $F_{1}\left(\alpha_{1}, \alpha_{2}\right)$ & $F_{2}\left(\alpha_{1}, \alpha_{2}\right)$ & $F\left(\alpha_{1}, \alpha_{2}\right)$ & $F(\alpha)$ \\
\hline$\beta$ & \multicolumn{5}{|c|}{ homogeneous impermeable wall conditions } \\
\hline 0.4 & 0.00104154 & 0.00104154 & 0.00208308 & 0.00208308 \\
\hline 0.8 & $9.94588 \mathrm{E}-4$ & $9.94588 \mathrm{E}-4$ & 0.00198918 & 0.00198918 \\
\hline 1.2 & $9.25165 \mathrm{E}-4$ & $9.25165 \mathrm{E}-4$ & 0.00185033 & 0.00185033 \\
\hline 1.6 & $8.42935 \mathrm{E}-4$ & $8.42935 \mathrm{E}-4$ & 0.00168587 & 0.00168587 \\
\hline 2.0 & $7.56654 \mathrm{E}-4$ & $7.56654 \mathrm{E}-4$ & 0.00151331 & 0.00151331 \\
\hline \hline$\beta$ & \multicolumn{5}{|c|}{ combined homogeneous and heterogeneous wall conditions } \\
\hline 2 & $8.48463 \mathrm{E}-4$ & $8.48463 \mathrm{E}-4$ & 0.001697 & 0.001697 \\
\hline 4 & $8.30289 \mathrm{E}-4$ & $8.30289 \mathrm{E}-4$ & 0.001661 & 0.001661 \\
\hline 6 & $8.22866 \mathrm{E}-4$ & $8.22866 \mathrm{E}-4$ & 0.001646 & 0.001646 \\
\hline 8 & $8.18832 \mathrm{E}-4$ & $8.18832 \mathrm{E}-4$ & 0.001638 & 0.001638 \\
\hline 10 & $8.16298 \mathrm{E}-4$ & $8.16298 \mathrm{E}-4$ & 0.001633 & 0.001633 \\
\hline & $Q_{1}$ & $Q_{2}$ & $Q$ & $Q$ \\
\hline
\end{tabular}

Table 3b. Values of volumetric flow rate for pure viscous fluid.

\begin{tabular}{|c|c|c|c|}
\hline \multicolumn{3}{|c|}{ Two fluid model (present model) } & $\begin{array}{c}\text { One fluid model } \\
\text { Gupta and Gupta (1972) }\end{array}$ \\
\hline$Q_{1}$ & $Q_{2}$ & $Q$ & $Q$ \\
\hline \multicolumn{4}{|c|}{ homogeneous impermeable wall conditions } \\
\hline$-9.62087 \mathrm{E}-4$ & $-9.62087 \mathrm{E}-4$ & -0.0019242 & -0.0019242 \\
\hline \multicolumn{3}{|c|}{ combined homogeneous and heterogeneous wall conditions } \\
\hline$-9.62087 \mathrm{E}-4$ & $-9.62087 \mathrm{E}-4$ & -0.0019242 & -0.00174605 \\
\hline
\end{tabular}

\section{Conclusions}

The problem of solute dispersion of a solute for composite porous medium between two parallel plates was studied using Taylor's dispersion model in the presence of first-order homogeneous and heterogeneous chemical reaction. Effective dispersion coefficient and volumetric flow rate were obtained for three different types of boundary conditions such as insulating, insulatingpermeable and permeable-insulating wall conditions for homogeneous chemical reaction and also for combined effect of homogeneous and heterogeneous chemical reactions.

The results obtained for insulating homogeneous first-order chemical reactions were:

1. As the homogeneous reaction rate parameter increases the effective dispersion coefficient decreases for all values of viscosity ratio, pressure gradients and porous parameter.

2. The effective dispersion coefficient increases as viscosity ratio and pressure gradient $(p>0$ and $p<0)$ increases.

3. The effect of porous parameter for soil, silica grains and wire cramps was to reduce the concentration which is the similar result obtained experimentally by Harleman et al. 1963. Further it was also observed that concentration distribution was high for air when compared to water.

The result obtained for homogeneous impermeable-permeable wall boundary conditions were:

1. As the homogeneous reaction rate parameter increases the effective dispersion coefficient decreases for any values of viscosity ratio, pressure gradient $(p>0)$ and porous parameter. For values of $p<0$, the effective dispersion coefficient increases as $\alpha$ increases.

2. The effective dispersion coefficient decreases as the viscosity ratio and pressure gradient increases whereas it increases as the porous parameter increases.

3. The effect of porous parameter on the concentration for soil, silica grains and wire cramps were not effective when compared to insulating wall boundary conditions. The distribution of concentration for water is less when compared to air.

The results obtained for homogeneous permeable-impermeable wall boundary conditions were:

1. The effect of viscosity ratio, pressure gradient and porous parameter on the homogeneous reaction rate parameter is similar to impermeable-permeable wall conditions. 
2. As the viscosity ratio increases $F$ also increases. The effect of effect of pressure gradient and porous parameter on $F$ is the similar result obtained for impermeable-permeable wall conditions.

3. The effect of porous parameter on the concentration for soil, silica grains and wire cramps were not effective when compared to insulating wall boundary conditions. The distribution of concentration for air is less when compared to water.

The results obtained for heterogeneous first-order chemical reactions were:

1. As the wall catalytic parameter increases the effective dispersion coefficient decreases for any value of viscosity ratio, pressure gradient and porous parameter.

2. The effective dispersion coefficient decreases as viscosity ratio $m$ and porous parameter $\sigma$ increases. The effective dispersion coefficient decreases for $p<1$ and increases for $p>1$ as pressure gradient $p$ increases.

3. The effect of porous parameter on the concentration for soil, silica grains and wire cramps for air and water showed the similar nature obtained for homogeneous insulating wall conditions.

The volumetric flow rate increases in magnitude as the viscosity ratio and porous parameter increases for small values and then remained invariant for large values for both homogeneous and heterogeneous wall boundary conditions.

The effective dispersion coefficient and volumetric flow rate for two fluid model in the presence of porous matrix (present model) agree very well with the results of Rudraiah and $\mathrm{Ng}$ (2007) for one fluid model. To further justify the results, the problem was also solved in the absence of porous parameter for two fluid model and the results were in good agreement with results of Gupta and Gupta (1972) for one fluid model.

\section{Nomenclature}

$C_{i} \quad$ concentration of the solute $\left(\mathrm{kg} \mathrm{m}^{-3}\right)$

$D_{i} \quad$ molecular diffusion coefficient

$D \quad$ ratio of molecular diffusion coefficient $\left(D_{2} / D_{1}\right)$

$h \quad$ distance between the plates $(\mathrm{m})$

$K_{i} \quad$ first-order reaction rate constant $\left(\mathrm{K}_{1} \mathrm{C}_{1} / \mathrm{mol} \mathrm{m}^{-3} \mathrm{~s}^{-1}\right)$

$L \quad$ typical length along the flow direction $(\mathrm{m})$

$Q_{i} \quad$ volumetric flow rate

$U_{i} \quad$ velocity $\left(\mathrm{ms}^{-1}\right)$

$\bar{u}_{i} \quad$ non-dimensional average velocity

$u_{i} \quad$ non-dimensional velocity

$\frac{d P_{i}}{d X_{i}} \quad$ pressure gradient $\left(\mathrm{Nm}^{-2}\right)$

$m \quad$ viscosity ratio $\left(\mu_{2} / \mu_{1}\right)$

$n \quad$ density ratio $\left(\rho_{1} / \rho_{2}\right)$

$p_{i} \quad$ non-dimensional pressure gradient

Greek symbols

$\eta \quad$ dimensionless length

$\alpha_{i}, \beta_{i}$ dimensionless reaction rate parameters

$\mu_{i} \quad$ dynamic viscosity $\left(\mathrm{kg} \mathrm{m}^{-1} \mathrm{~s}^{-1}\right)$

$\kappa_{i} \quad$ permeability of the porous medium $\left(\mathrm{m}^{2}\right)$

$\sigma_{i} \quad$ porous parameter $\left(h / \sqrt{\kappa_{i}}\right)$

$\rho_{i} \quad$ density of the fluid $\left(\mathrm{kg} \mathrm{m}^{-3}\right)$

Subscripts

$i=1,2$ where 1,2 -quantities for region- 1 and region-2, respectively. 


\section{Appendix}

\section{Case 1: Diffusion with a homogeneous first-order chemical reaction}

$u_{1}=a_{1} \cosh \sigma_{1} \eta+a_{2} \sinh \sigma_{1} \eta-\frac{p_{1}}{\sigma_{1}^{2}} ; u_{2}=a_{3} \cosh \sigma_{2} \eta+a_{4} \sinh \sigma_{2} \eta-\frac{p_{2}}{\sigma_{2}^{2}} ;$

$a_{4}=\frac{\sigma_{1}\left(\cosh \sigma_{1} \cosh \sigma_{2}\left(\frac{p_{1}}{\sigma_{1}^{2}}-\frac{m n p_{2}}{\sigma_{2}{ }^{2}}\right)+\frac{p_{2} m n}{\sigma_{2}^{2}} \cosh \sigma_{1}-\frac{p_{1}}{\sigma_{1}^{2}} \cosh \sigma_{2}\right)}{m n \sigma_{1} \cosh \sigma_{1} \sinh \sigma_{2}+m^{2} n \sigma_{2} \sinh \sigma_{1} \cosh \sigma_{2}} ; a_{3}=\frac{1}{\cosh \sigma_{2}}\left(-a_{4} \sinh \sigma_{2}+\frac{p_{2}}{\sigma_{2}^{2}}\right) ; \quad a_{2}=\frac{m^{2} n \sigma_{2}}{\sigma_{1}} a_{4} ;$

$a_{1}=\frac{1}{\cosh \sigma_{1}}\left(a_{2} \sinh \left(\sigma_{1}\right)+\frac{p_{1}}{\sigma_{1}^{2}}\right) ; l_{1}=\frac{-p_{1}}{\sigma_{1}^{2}}-\left(\bar{u}_{1}+\bar{u}_{2}\right) ; \quad l_{2}=\frac{-p_{2}}{\sigma_{2}^{2}}-\left(\bar{u}_{1}+\bar{u}_{2}\right)$.

Concentration distribution with impermeable wall conditions

$g_{1}=-\frac{a_{1} \sigma_{1} \sinh \sigma_{1}}{\sigma_{1}^{2}-\alpha_{1}^{2}}+\frac{a_{2} \sigma_{1} \cosh \sigma_{1}}{\sigma_{1}^{2}-\alpha_{1}^{2}} ; \quad g_{2}=\frac{a_{3} \sigma_{2} \sinh \sigma_{2}}{\sigma_{2}^{2}-\alpha_{2}^{2}}+\frac{a_{4} \sigma_{2} \cosh \sigma_{2}}{\sigma_{2}^{2}-\alpha_{2}^{2}} ; \quad g_{3}=\frac{a_{1}}{\sigma_{1}^{2}-\alpha_{1}^{2}}-\frac{l_{1}}{\alpha_{1}^{2}} ; \quad g_{4}=-\left(\frac{a_{3}}{\sigma_{2}^{2}-\alpha_{2}^{2}}-\frac{l_{2}}{\alpha_{2}^{2}}\right) ;$

$g_{5}=\frac{a_{2} \sigma_{1}}{\sigma_{1}^{2}-\alpha_{1}^{2}} ; \quad g_{6}=-\frac{D \sigma_{2} a_{4}}{\sigma_{2}^{2}-\alpha_{2}^{2}} ; Z_{1}=\frac{h^{2}}{D_{1} L} \frac{\partial C_{1}}{\partial \xi_{1}} ; Z_{2}=\frac{h^{2}}{D_{2} L} \frac{\partial C_{2}}{\partial \xi_{2}}$

$b_{1}=Z_{1} b_{11}+Z_{2} b_{12} ; b_{2}=Z_{1} b_{21}+Z_{2} b_{22} ; b_{3}=Z_{1} b_{31}+Z_{2} b_{32} ; b_{4}=Z_{1} b_{41}+Z_{2} b_{42}$;

$\operatorname{Dr}=\alpha_{1} \alpha_{2} \sinh \left(\alpha_{1}\right) \cosh \left(\alpha_{2}\right)+\alpha_{2}^{2} D \sinh \left(\alpha_{2}\right) \cosh \left(\alpha_{1}\right)$;

$b_{41}=\frac{-1}{\mathrm{Dr}}\left(g_{3} \alpha_{1} \alpha_{2} \sinh \left(\alpha_{1}\right) \sinh \left(\alpha_{2}\right)-g_{5} \alpha_{2} \cosh \left(\alpha_{1}\right) \sinh \left(\alpha_{2}\right)+g_{1} \alpha_{2} \sinh \left(\alpha_{2}\right)\right)$;

$b_{42}=\frac{-1}{\operatorname{Dr}}\left(g_{2} \alpha_{1} \sinh \left(\alpha_{2}\right)+g_{4} \alpha_{1} \alpha_{2} \sinh \left(\alpha_{1}\right) \sinh \left(\alpha_{2}\right)-g_{6} \alpha_{2} \cosh \left(\alpha_{1}\right) \sinh \left(\alpha_{2}\right)\right)$;

$b_{31}=\frac{-b_{41} \cosh \left(\alpha_{2}\right)}{\sinh \left(\alpha_{2}\right)} ; b_{32}=\frac{-b_{42} \alpha_{2} \cosh \left(\alpha_{2}\right)-g_{2}}{\alpha_{2} \sinh \left(\alpha_{2}\right)} ; b_{11}=b_{31}-g_{3} ; b_{12}=b_{32}-g_{4} ; b_{21}=\frac{b_{11} \alpha_{1} \sinh \left(\alpha_{1}\right)-g_{1}}{\alpha_{1} \cosh \left(\alpha_{1}\right)} ; b_{22}=\frac{b_{12} \sinh \left(\alpha_{1}\right)}{\cosh \left(\alpha_{1}\right)}$.

Concentration distribution with lower wall impermeable and upper wall permeable wall conditions.

$g_{1}=-\frac{a_{1} \sigma_{1} \sinh \sigma_{1}}{\sigma_{1}^{2}-\alpha_{1}^{2}}+\frac{a_{2} \sigma_{1} \cosh \sigma_{1}}{\sigma_{1}^{2}-\alpha_{1}^{2}} ; g_{2}=\frac{a_{3} \cosh \sigma_{2}}{\sigma_{2}^{2}-\alpha_{2}^{2}}+\frac{a_{4} \sinh \sigma_{2}}{\sigma_{2}^{2}-\alpha_{2}^{2}}-\frac{l_{2}}{\alpha_{2}^{2}} ; g_{3}=\frac{a_{1}}{\sigma_{1}^{2}-\alpha_{1}^{2}}-\frac{l_{1}}{\alpha_{1}^{2}} ; \quad g_{4}=-\left(\frac{a_{3}}{\sigma_{2}^{2}-\alpha_{2}^{2}}-\frac{l_{2}}{\alpha_{2}^{2}}\right)$;

$g_{5}=\frac{a_{2} \sigma_{1}}{\sigma_{1}^{2}-\alpha_{1}^{2}} ; \quad g_{6}=-\frac{D \sigma_{2} a_{4}}{\sigma_{2}^{2}-\alpha_{2}^{2}} ; Z_{1}=\frac{h^{2}}{D_{1} L} \frac{\partial C_{1}}{\partial \xi_{1}} ; Z_{2}=\frac{h^{2}}{D_{2} L} \frac{\partial C_{2}}{\partial \xi_{2}} ; \operatorname{Dr}=\alpha_{1} \sinh \left(\alpha_{1}\right) \sinh \left(\alpha_{2}\right)+\alpha_{2} D \cosh \left(\alpha_{1}\right) \cosh \left(\alpha_{2}\right)$;

$b_{1}=Z_{1} b_{11}+Z_{2} b_{12}+b_{1 c} ; b_{2}=Z_{1} b_{21}+Z_{2} b_{22}+b_{2 c} ; b_{3}=Z_{1} b_{31}+Z_{2} b_{32}+b_{3 c} ; b_{4}=Z_{1} b_{41}+Z_{2} b_{42}+b_{4 c} ;$

$b_{41}=\frac{-1}{\operatorname{Dr}}\left(g_{3} \alpha_{1} \sinh \left(\alpha_{1}\right) \cosh \left(\alpha_{2}\right)-g_{5} \cosh \left(\alpha_{1}\right) \cosh \left(\alpha_{2}\right)+g_{1} \cosh \left(\alpha_{2}\right)\right)$;

$b_{42}=\frac{-1}{\operatorname{Dr}}\left(g_{2} \alpha_{1} \sinh \left(\alpha_{1}\right)+g_{4} \alpha_{1} \sinh \left(\alpha_{1}\right) \cosh \left(\alpha_{2}\right)-g_{6} \cosh \left(\alpha_{1}\right) \cosh \left(\alpha_{2}\right)\right) ; b_{4 c}=\frac{\alpha_{1} \sinh \left(\alpha_{1}\right)}{\operatorname{Dr}} ; b_{31}=\frac{-b_{41} \sinh \left(\alpha_{2}\right)}{\cosh \left(\alpha_{2}\right)}$;

$b_{32}=\frac{-b_{42} \sinh \left(\alpha_{2}\right)-g_{2}}{\cosh \left(\alpha_{2}\right)} ; b_{3 c}=\frac{-b_{4 c} \sinh \left(\alpha_{2}\right)+1}{\cosh \left(\alpha_{2}\right)} ; b_{11}=b_{31}-g_{3} ; b_{12}=b_{32}-g_{4} ; b_{1 c}=b_{3 c} ; b_{21}=\frac{b_{11} \alpha_{1} \sinh \left(\alpha_{1}\right)-g_{1}}{\alpha_{1} \cosh \left(\alpha_{1}\right)}$;

$b_{22}=\frac{b_{12} \sinh \left(\alpha_{1}\right)}{\cosh \left(\alpha_{1}\right)} ; b_{2 c}=\frac{b_{1 c} \sinh \left(\alpha_{1}\right)}{\cosh \left(\alpha_{1}\right)}$.

Concentration distribution with lower wall permeable and upper wall impermeable wall conditions.

$g_{1}=\frac{a_{1} \cosh \sigma_{1}}{\sigma_{1}^{2}-\alpha_{1}^{2}}-\frac{a_{2} \sinh \sigma_{1}}{\sigma_{1}^{2}-\alpha_{1}^{2}}-\frac{l_{1}}{\alpha_{1}^{2}} ; g_{2}=\frac{a_{3} \sigma_{2} \sinh \sigma_{2}}{\sigma_{2}^{2}-\alpha_{2}^{2}}+\frac{a_{4} \sigma_{2} \cosh \sigma_{2}}{\sigma_{2}^{2}-\alpha_{2}^{2}} ; g_{3}=\frac{a_{1}}{\sigma_{1}^{2}-\alpha_{1}^{2}}-\frac{l_{1}}{\alpha_{1}^{2}} ; \quad g_{4}=-\left(\frac{a_{3}}{\sigma_{2}^{2}-\alpha_{2}^{2}}-\frac{l_{2}}{\alpha_{2}^{2}}\right)$;

$g_{5}=\frac{a_{2} \sigma_{1}}{\sigma_{1}^{2}-\alpha_{1}^{2}} ; g_{6}=-\frac{D \sigma_{2} a_{4}}{\sigma_{2}^{2}-\alpha_{2}^{2}} ; Z_{1}=\frac{h^{2}}{D_{1} L} \frac{\partial C_{1}}{\partial \xi_{1}} ; Z_{2}=\frac{h^{2}}{D_{2} L} \frac{\partial C_{2}}{\partial \xi_{2}} ; \operatorname{Dr}=\alpha_{2} \cosh \left(\alpha_{2}\right) \cosh \left(\alpha_{1}\right)+D \alpha_{2}^{2} \sinh \left(\alpha_{2}\right) \sinh \left(\alpha_{1}\right)$;

$b_{1}=Z_{1} b_{11}+Z_{2} b_{12}+b_{1 c} ; b_{2}=Z_{1} b_{21}+Z_{2} b_{22}+b_{2 c} ; b_{3}=Z_{1} b_{31}+Z_{2} b_{32}+b_{3 c} ; b_{4}=Z_{1} b_{41}+Z_{2} b_{42}+b_{4 c}$;

$b_{41}=\frac{-1}{\operatorname{Dr}}\left(g_{3} \alpha_{2} \cosh \left(\alpha_{1}\right) \sinh \left(\alpha_{2}\right)-g_{5} \frac{\alpha_{2}}{\alpha_{1}} \sinh \left(\alpha_{2}\right) \sinh \left(\alpha_{1}\right)-g_{1} \alpha_{2} \sinh \left(\alpha_{2}\right)\right)$; 


$$
\begin{aligned}
& b_{42}=\frac{-1}{\operatorname{Dr}}\left(g_{2} \cosh \left(\alpha_{1}\right)-g_{4} \alpha_{2} \cosh \left(\alpha_{1}\right) \sinh \left(\alpha_{2}\right)-g_{6} \frac{\alpha_{2}}{\alpha_{1}} \sinh \left(\alpha_{2}\right) \sinh \left(\alpha_{1}\right)\right) ; b_{4 c}=\frac{-\alpha_{2} \sinh \left(\alpha_{2}\right)}{\operatorname{Dr}} ; b_{31}=\frac{-b_{41} \cosh \left(\alpha_{2}\right)}{\sinh \left(\alpha_{2}\right)} ; \\
& b_{32}=\frac{-b_{42} \alpha_{2} \cosh \left(\alpha_{2}\right)-g_{2}}{\alpha_{2} \sinh \left(\alpha_{2}\right)} ; b_{3 c}=\frac{-b_{4 c}}{\alpha_{2} \sinh \left(\alpha_{2}\right)} ; b_{11}=b_{31}-g_{3} ; b_{12}=b_{32}-g_{4} ; b_{1 c}=b_{3 c} ; b_{21}=\frac{b_{11} \cosh \left(\alpha_{1}\right)+g_{1}}{\sinh \left(\alpha_{1}\right)} ; \\
& b_{22}=\frac{b_{12} \cosh \left(\alpha_{1}\right)}{\sinh \left(\alpha_{1}\right)} ; b_{2 c}=\frac{b_{1 c} \cosh \left(\alpha_{1}\right)-1}{\sinh \left(\alpha_{1}\right)} .
\end{aligned}
$$

\section{Case 2 Diffusion with combined homogeneous and heterogeneous first-order chemical reaction}

$$
\begin{aligned}
& g_{1}=-\frac{a_{1} \sigma_{1} \sinh \sigma_{1}}{\sigma_{1}^{2}-\alpha_{1}^{2}}+\frac{a_{2} \sigma_{1} \cosh \sigma_{1}}{\sigma_{1}^{2}-\alpha_{1}^{2}} ; g_{2}=\frac{a_{3} \sigma_{2} \sinh \sigma_{2}}{\sigma_{2}^{2}-\alpha_{2}^{2}}+\frac{a_{4} \sigma_{2} \cosh \sigma_{2}}{\sigma_{2}^{2}-\alpha_{2}^{2}} ; g_{3}=\frac{a_{1}}{\sigma_{1}^{2}-\alpha_{1}^{2}}-\frac{l_{1}}{\alpha_{1}^{2}} ; \quad g_{4}=-\left(\frac{a_{3}}{\sigma_{2}^{2}-\alpha_{2}^{2}}-\frac{l_{2}}{\alpha_{2}^{2}}\right) ; \\
& g_{5}=\frac{a_{2} \sigma_{1}}{\sigma_{1}^{2}-\alpha_{1}^{2}} ; \quad g_{6}=-\frac{D \sigma_{2} a_{4}}{\sigma_{2}^{2}-\alpha_{2}^{2}} ; Z_{1}=\frac{h^{2}}{D_{1} L} \frac{\partial C_{1}}{\partial \xi_{1}} ; Z_{2}=\frac{h^{2}}{D_{2} L} \frac{\partial C_{2}}{\partial \xi_{2}} ; f_{1}=g_{1}-\beta_{1}\left(\frac{a_{1} \cosh \left(\sigma_{1}\right)}{\sigma_{1}^{2}-\alpha_{1}^{2}}-\frac{a_{2} \sinh \left(\sigma_{1}\right)}{\sigma_{1}^{2}-\alpha_{1}^{2}}-\frac{l_{1}}{\alpha_{1}^{2}}\right) ; \\
& f_{2}=g_{2}+\beta_{2}\left(\frac{a_{3} \cosh \left(\sigma_{2}\right)}{\sigma_{2}^{2}-\alpha_{2}^{2}}+\frac{a_{4} \sinh \left(\sigma_{2}\right)}{\sigma_{2}^{2}-\alpha_{2}^{2}}-\frac{l_{2}}{\alpha_{2}^{2}}\right) ; Z_{1}=\frac{h^{2}}{D_{1} L} \frac{\partial C_{1}}{\partial \xi_{1}} ; Z_{2}=\frac{h^{2}}{D_{2} L} \frac{\partial C_{2}}{\partial \xi_{2}} ; b_{1}=Z_{1} b_{11}+Z_{2} b_{12} ; b_{2}=Z_{1} b_{21}+Z_{2} b_{22} \text {; } \\
& b_{3}=Z_{1} b_{31}+Z_{2} b_{32} ; b_{4}=Z_{1} b_{41}+Z_{2} b_{42} \text {; } \\
& \operatorname{Dr}=\left(\alpha_{2} \cosh \left(\alpha_{2}\right)+\beta_{2} \sinh \left(\alpha_{2}\right)\right)\left(\alpha_{1} \sinh \left(\alpha_{1}\right)+\beta_{1} \cosh \left(\alpha_{1}\right)\right)+\frac{\alpha_{2} D}{\alpha_{1}}\left(\alpha_{1} \cosh \left(\alpha_{1}\right)+\beta_{1} \sinh \left(\alpha_{1}\right)\right)\left(\alpha_{2} \sinh \left(\alpha_{2}\right)+\beta_{2} \cosh \left(\alpha_{2}\right)\right) \text {; } \\
& b_{41}=\frac{-1}{\operatorname{Dr}}\left(g_{3}\left(\alpha_{1} \sinh \left(\alpha_{1}\right)+\beta_{1} \cosh \left(\alpha_{1}\right)\right)-\frac{g_{5}}{\alpha_{1}}\left(\alpha_{1} \cosh \left(\alpha_{1}\right)+\beta_{1} \sinh \left(\alpha_{1}\right)\right)+f_{1}\right)\left(\alpha_{2} \sinh \left(\alpha_{2}\right)+\beta_{2} \cosh \left(\alpha_{2}\right)\right) ; \\
& b_{42}=\frac{-1}{\operatorname{Dr}}\left(f_{2}+\left(g_{4}-\frac{g_{6}}{\alpha_{1}}\right)\left(\alpha_{2} \sinh \left(\alpha_{2}\right)+\beta_{2} \cosh \left(\alpha_{2}\right)\right)\right)\left(\alpha_{1} \sinh \left(\alpha_{1}\right)+\beta_{1} \cosh \left(\alpha_{1}\right)\right) ; b_{31}=\frac{-b_{41}\left(\alpha_{2} \cosh \left(\alpha_{2}\right)+\beta_{2} \sinh \left(\alpha_{2}\right)\right)}{\left(\alpha_{2} \sinh \left(\alpha_{2}\right)+\beta_{2} \cosh \left(\alpha_{2}\right)\right)} \text {; } \\
& b_{32}=\frac{-b_{42}\left(\alpha_{2} \cosh \left(\alpha_{2}\right)+\beta_{2} \sinh \left(\alpha_{2}\right)\right)-f_{2}}{\left(\alpha_{2} \sinh \left(\alpha_{2}\right)+\beta_{2} \cosh \left(\alpha_{2}\right)\right)} ; b_{11}=b_{31}-g_{3} ; b_{12}=b_{32}-g_{4} ; b_{21}=\frac{b_{11}\left(\alpha_{1} \sinh \left(\alpha_{1}\right)+\beta_{1} \cosh \left(\alpha_{1}\right)\right)-f_{1}}{\left(\alpha_{1} \cosh \left(\alpha_{1}\right)+\beta_{1} \sinh \left(\alpha_{1}\right)\right)} ; \\
& b_{22}=\frac{b_{12}\left(\alpha_{1} \sinh \left(\alpha_{1}\right)+\beta_{1} \cosh \left(\alpha_{1}\right)\right)}{\left(\alpha_{1} \cosh \left(\alpha_{1}\right)+\beta_{1} \sinh \left(\alpha_{1}\right)\right)} \text {. }
\end{aligned}
$$

Case 3: The channel filled with porous matrix (one fluid model)

Concentration distribution with homogeneous impermeable wall conditions

$$
l_{1}=\frac{p}{\sigma^{2}\left(\sigma^{2}-\alpha^{2}\right) \cosh (\sigma)} ; l_{2}=\frac{p \tanh (\sigma)}{\sigma^{3} \alpha^{2}} ; b_{1}=-\frac{l_{1} \sigma \sinh (\sigma)}{\alpha \sinh (\alpha)} \text {. }
$$

Concentration distribution with lower wall impermeable and upper wall permeable wall conditions.

$$
\begin{aligned}
& l_{1}=\frac{p}{\sigma^{2}\left(\sigma^{2}-\alpha^{2}\right) \cosh (\sigma)} ; l_{2}=\frac{p \tanh (\sigma)}{\sigma^{3} \alpha^{2}} ; b_{1}=\frac{b_{2} \alpha \cosh (\alpha)-l_{1} z \sigma \sinh (\sigma)}{\alpha \sinh (\alpha)} ; \\
& b_{2}=-\frac{l_{1} z(\alpha \cosh (\sigma) \sinh (\alpha)-\sigma \sinh (\sigma) \cosh (\alpha))+\left(l_{2} z-1\right) \alpha \sinh (\alpha)}{\alpha \cosh (2 \alpha)} .
\end{aligned}
$$

Concentration distribution with lower wall permeable and upper wall impermeable wall conditions.

$$
\begin{aligned}
& l_{1}=\frac{p}{\sigma^{2}\left(\sigma^{2}-\alpha^{2}\right) \cosh (\sigma)} ; l_{2}=\frac{p \tanh (\sigma)}{\sigma^{3} \alpha^{2}} ; b_{1}=\frac{b_{2} \sinh (\alpha)-l_{1} z \cosh (\sigma)-l_{2} z+1}{\cosh (\alpha)} ; \\
& b_{2}=\frac{\left(l_{1} z \cosh (\sigma)+l_{2} z-1\right) \alpha \sinh (\alpha)-l_{1} z \sigma \sinh (\sigma) \cosh (\alpha)}{\alpha \cosh (2 \alpha)} ; Z=\frac{h^{2}}{D L} \frac{\partial C}{\partial \xi} .
\end{aligned}
$$

Concentration distribution with heterogeneous impermeable wall conditions

$$
l_{1}=\frac{p}{\sigma^{2}\left(\sigma^{2}-\alpha^{2}\right) \cosh (\sigma)} ; l_{2}=\frac{p \tanh (\sigma)}{\sigma^{3} \alpha^{2}} ; b_{1}=\frac{-l_{1} z \sigma \sinh (\sigma)-\beta\left(l_{1} z \cosh (\sigma) l_{2} z\right)}{\alpha \sinh (\alpha)+\beta \cosh (\alpha)} ; b_{2}=0 \text {. }
$$


Case 4a: Effect of homogeneous and heterogeneous reactions on the dispersion of solute in the absence of porous matrix (two fluid model).

$$
\begin{aligned}
& a_{1}=-a_{4}+\frac{p_{2}}{2} ; a_{2}=m n a_{4} ; a_{3}=-a_{4}-\frac{p_{2}}{2} ; a_{4}=\frac{-p_{1}}{2 m n(m+1)}-\frac{-p_{2} m}{2(m+1)} ; l_{1}=-\frac{1}{2}\left(\frac{p_{1}}{6}-\frac{a_{1}}{2}-a_{2}+\frac{p_{2}}{6}+\frac{a_{3}}{2}+a_{4}\right) ; \\
& l_{2}=-\frac{1}{2}\left(\frac{p_{1}}{6}-\frac{a_{1}}{2}+a_{2}+\frac{p_{2}}{6}+\frac{a_{3}}{2}-a_{4}\right) ; l_{1}=\frac{-p_{1}}{2 \alpha_{1}^{2}} ; l_{2}=\frac{-a_{1}}{\alpha_{1}^{2}} ; l_{3}=-\frac{l c_{1}}{\alpha_{1}^{2}}-\frac{p_{1}}{\alpha_{1}^{4}} ; l_{4}=\frac{-p_{2}}{2 \alpha_{2}^{2}} ; l_{5}=\frac{-a_{3}}{\alpha_{2}^{2}} ; l_{6}=-\frac{l c_{2}}{\alpha_{2}^{2}}-\frac{p_{2}}{\alpha_{2}^{4}} ; \\
& Z_{1}=\frac{h^{2}}{D_{1} L} \frac{\partial C_{1}}{\partial \xi_{1}} ; Z_{2}=\frac{h^{2}}{D_{2} L} \frac{\partial C_{2}}{\partial \xi_{2}} ; b_{1}=Z_{1} b_{11}+Z_{2} b_{12} ; b_{2}=Z_{1} b_{21}+Z_{2} b_{22} ; b_{3}=Z_{1} b_{31}+Z_{2} b_{32} ; b_{4}=Z_{1} b_{41}+Z_{2} b_{42} ; \\
& \operatorname{Dr}=\alpha_{1} \alpha_{2} \sinh \left(\alpha_{1}\right) \cosh \left(\alpha_{2}\right)+\alpha_{2}^{2} D \sinh \left(\alpha_{2}\right) \cosh \left(\alpha_{1}\right) ; \\
& b_{41}=\frac{-1}{\operatorname{Dr}}\left(l_{3} \alpha_{1} \alpha_{2} \sinh \left(\alpha_{1}\right) \sinh \left(\alpha_{2}\right)-l_{2} \alpha_{2} \cosh \left(\alpha_{1}\right) \sinh \left(\alpha_{2}\right)+\alpha_{2} \sinh \left(\alpha_{2}\right)\left(l_{2}-2 l_{1}\right)\right) ; \\
& b_{42}=\frac{-1}{\operatorname{Dr}}\left(\alpha_{1} \sinh \left(\alpha_{2}\right)\left(l_{5}+2 l_{4}\right)-l_{6} \alpha_{1} \alpha_{2} \sinh \left(\alpha_{1}\right) \sinh \left(\alpha_{2}\right)+l_{5} D \alpha_{2} \cosh \left(\alpha_{1}\right) \sinh \left(\alpha_{2}\right)\right) ; \\
& b_{31}=\frac{-b_{41} \cosh \left(\alpha_{2}\right)}{\sinh \left(\alpha_{2}\right)} ; b_{32}=\frac{-b_{42} \alpha_{2} \cosh \left(\alpha_{2}\right)-2 l_{4}-l_{5}}{\alpha_{2} \sinh \left(\alpha_{2}\right)} ; b_{11}=b_{31}-l_{3} ; b_{12}=b_{32}+l_{6} ; b_{21}=\frac{b_{11} \alpha_{1} \sinh \left(\alpha_{1}\right)+2 l_{1}-l_{2}}{\alpha_{1} \cosh \left(\alpha_{1}\right)} \\
& b_{22}=\frac{b_{12} \sinh \left(\alpha_{1}\right)}{\cosh \left(\alpha_{1}\right)}
\end{aligned}
$$

Acknowledgement: One of the authors (JCU) thank UGC-New Delhi for the financial support under UGC-Major Research Project.

\section{References}

Abdel-Rahman, G.M., 2008. Thermal diffusion and MHD effects on combined free-forced convection and mass transfer of a viscous fluid flow through a porous medium with heat generation, Chem. Eng. Technol., Vol. 31, No. 4, pp. 554-559.

Al-Niami, A.N.S., and Rushton, K.R., 1977. Analysis of flow against dispersion in porous media, J. Hydrol., Vol. 33, pp. 87-97.

Alzami, B., and Vafai, K., 2001. Analysis of fluid flow and heat transfer interfacial conditions between a porous medium and a fluid layer, Int. J. Heat Mass Transfer, Vol. 44, pp. 1735-1749.

Beavers, G.S., and Joseph, D.D., 1967. Boundary conditions at a naturally permeable wall, J. Fluid Mech., Vol. 30, pp. 197-207.

Beckermann, C., Viskanta, R., and Ramadhyani, S., 1987. Natural convection flow and heat transfer between a fluid layer and a porous layer inside a rectangular enclosure, ASME, J. Heat Transfer, Vol. 109, pp. 363-370.

Beckermann, C., Viskanta, R., and Ramadhyani, S., 1988. Natural convection in vertical enclosures containing simultaneously fluid and porous layers, J. Fluid Mech., Vol. 186, pp. 257-284.

Bird, R.B., Stewart, W.E., and Lightfoot, E.N., 1960. Transport phenomena, John Wiley \& Sons, New York, pp. 542-546.

Boming Yu, and Wei Liu, 2003. Fractal analysis of permeabilities for porous media, AIChE J., Vol. 50(1), pp. 46-57.

Boming Yu, 2008. Analysis of flow in fractal porous media, Applied Mechanics Reviews, Vol. 61(5), pp. 050801.

Bruce, J.C., and Street, R.L., 1966. Studies of free surface flow and two dimensional dispersion in porous media, Civil Eng. Deptt. Stanford Univ., Stanford, California, Report No. 63.

Cussler, E.L., 1998. Diffusion mass transfer in fluid systems, Camb. Univ. Press.

Gupta, P.S. and Gupta, A.S., 1972. Effect of homogeneous and heterogeneous reactions on the dispersion of solute in a laminar flow between two plates, Proc. Roy. Soc. Lond. A, Vol. 330, pp. 59-63.

Harlemann, D.R.F., Melhorn, P.F., Rumer, R.R., 1963. Dispersion-permeability correlation in porous media, J. Hydraulic Div. Am. Soc. Civil Engrs., Vol. 89, pp. 67.

Hoopes, J.A., and Harteman, D.R.F., 1965. Waste water recharge and dispersion in porous media, Technical Report No. 75.

Katz, A. J., and Thompson, A. H., 1985. Fractal sandstone pores: Implications for conductivity and pore formation, Phys. Rev. Lett., Vol. 54, pp. 1325.

Kaviany, M., 1999. Principals of heat transfer in porous media, Springer New York.

Malashetty, M.S., Umavathi, J.C. and Prathap Kumar, J., 2001. Convective flow and heat transfer in a composite porous medium, J. Porous Media, Vol. 4, No. 1, pp. 15-22.

Malashetty, M.S., Umavathi, J.C. and Prathap Kumar, J., 2005. Flow and heat transfer in an inclined channel containing a fluid layer sandwiched between two porous layers, J. Porous Media, Vol. 8, No. 1, pp. 443-453.

Marino, M.A., 1974. Distribution of contaminants in porous media flow, Water Resour. Res., Vol. 10, No. 5.

Marino, M.A., 1978. Flow against dispersion in nonadsorbing porous media, J. Hydrol., Vol. 37, pp. 149-158.

Neale, G., and Nader, W., 1974. Practical significance of Brinkman's extension of Darcy's law: coupled parallel flows within a channel and a bounding porous medium, Can. J. Chem. Eng., Vol. 52, pp. 475-478. 
Ng, C.O., Rudraiah, N., and Nagaraja, H.N., 2005. Electrohydrodynamic dispersion of macro molecular components in nanostructured biological bearing, J. Energy Heat Mass Transfer, Vol. 27, pp. 39-64.

Panfilov, M., (2000). Macroscale models of flow through highly heterogeneous porous media, Kluwer, London.

Poulikakos, D., Bejan, A. Selimos, B., and Blake, K.R., 1986. High Rayleigh number convection in a fluid overlying a porous bed, Int. J. Heat Fluid Flow, Vol. 7, pp. 109-116.

Prasad, V., 1991. Convective flow interaction and heat transfer between fluid and porous layers, in: Proceedings of NATO, ASI convective Heat and Mass Transfer in Porous Medium, Izmir, Turkey, pp. 563-615.

Prathap Kumar, J., Umavathi, J.C. and Basavaraj M Biradar, 2010. Mixed convection of composite porous medium in a vertical channel with asymmetric wall heating conditions, J. Porous Media, Vol. 13, No. 3, pp. 271-285.

Prathap Kumar, J., Umavathi, J.C., Pop, I. and Basavaraj M Biradar, 2009. Fully developed mixed convection flow in a vertical channel containing porous and fluid layers with isothermal or isoflux boundaries, Trans. Porous Media, Vol. 80, pp. 117-135.

Prathap Kumar, J., Umavathi, J.C., and Shivakumar Madhavarao, 2011. Dispersion in composite porous medium with homogeneous and heterogeneous chemical reactions, Heat Transfer-Asian Research, Vol. 40 (7), pp. 608-640.

Rudraiah, N. and Chiu-On-Ng., 2007. Dispersion in porous media with and without reaction: A review, J. Porous Media, Vol. 10, No. 3, pp. 219-248.

Solomon, R.L. and Hudson, J.L., 1967. Heterogeneous and homogeneous reactions in a tubular reactor, AIChE. Jl., Vol. 13, No. 3, pp. 545-550.

Sueiu, A.N., Iwatsubo, T., and Matsuda, M., 2003. Theoretical investigation of an artificial joint with micro-pocket-covered component and biphatic cartilage on the opposite articulate surface, ASME J. Biomech. Eng., Vol. 125, pp. 425-433.

Taylor, G.I., 1953. Dispersion of a solute in a solvent under laminar conditions, Proc. Roy. Soc. Lond. A, Vol. 219, pp. 186-203.

Umavathi, J.C., Chamkha, A.J. and Sridhar, K.S.R., 2010. Generalized plain Couette flow heat transfer in a composite channel, Trans. Porous Media, Vol. 85, pp. 157-169.

Umavathi, J.C., Chamkha, A.J., Abdul Mateen and Al-Mudhaf, A., 2006. Oscillatory flow and heat transfer in a horizontal composite porous medium channel, Int. J. Heat and Technology, Vol. 24, pp. 75-86.

Umavathi, J.C., Chamkha, A.J., Abdul Mateen and Al-Mudhaf, A., 2009. Unsteady oscillatory flow and heat transfer in a horizontal composite porous medium channel, Nonlinear Analysis Modelling and Control, Vol. 14, pp. 1-19.

Vafai, K., 2000. Handbook of porous media, Marcel Dekker New York.

Vafai, K., and Kim, S.J., 1990. Fluid mechanics of the interface region between a porous medium and a fluid layer-an exact solution, Int. J. Heat Fluid Flow, Vol. 11, pp. 254-256.

Walker, R.E., 1961. Chemical reaction and diffusion in a catalytic tubular reactor, Phys. Fluids, Vol. 4, No.10, pp. 1211-1216.

Wooding, R.A., 1960. Instability of a viscous liquid of variable density in a vertical Hele-Shaw cell, J. Fluid Mech., Vol. 7, pp. 501-515.

Yongting Ma, Boming Yu, Duanming Zhang and Mingqing Zou, 2003. A self-similarity model for effective thermal conductivity of porous media, J. Physics D: Applied Physics, Vol. 36, No. 17, pp. 2157-2164.

Yu, B. M., Lee, L.J. and Cao, H.Q., 2001. Fractal characters of pore microstructures of textile fabrics, Fractals, Vol. 9, pp. 155.

Yu, B. M., Lee, L.J. and Cao, H.Q., 2002. A fractal in-plane permeability model for fabrics, Polym. Compos., Vol. 23, No. 2, pp. 201.

Yu, B. M., and Cheng, P., 2002. A fractal permeability model for bi-dispersed porous media, Int. J. Heat Mass Transfer, Vol. 45, No. 14, pp. 2983.

\section{Biographical notes}

J. Prathap Kumar received M. Phil. degree from Mangalore University Mangalore in 1990 and Ph. D degree from Gulbarga University Gulbarga India in 2003. $\mathrm{He}$ is an associate professor in the department of Mathematics Gulbarga University, Gulbarga, Karnataka, India. His research interest includes heat and mass transfer, dispersion, baffles for flow through various geometries of one and two fluids models. He has published more than 30 papers in referred International journals. He has also presented 10 research articles in National and International Conferences.

J. C. Umavathi received Ph. D degree from Gulbarga University Gulbarga India in 1992. She is a Professor in the department of Mathematics, Gulbarga University, Gulbarga, Karnataka, India. Her research interest includes heat and mass transfer of multiple (Newtonian and non-Newtonian) fluids through channels and rectangular ducts, numerical simulation using Finite differences and Range-Kutta Gill method, magnetohydradynamics, flow through porous media. She has published more than 80 papers in referred International journals. She has also presented more than 25 research articles in National and International conferences. She is currently dealing with few projects sponsored by Government of India.

Shivakumar Madhavarao received Master of Philosophy in Mathematics from Gulbarga University, Gulbarga, Karnataka, India in 2007. He is working for Doctoral degree. His research interest includes dispersion, two fluid flows for Newtonian fluids through channels.

Received April 2012

Accepted May 2012

Final acceptance in revised form June 2012 